\title{
Psychoanalytische Psychotherapie mit Muslim*innen der Schweiz
}

\section{Innerpsychische Konfliktlagen im Kontext muslimischer Religiosität}

\author{
Katrin Hartmann \\ Psychotherapie-Wissenschaft 9 (1) 23-37 2019 \\ www.psychotherapie-wissenschaft.info \\ CC BY-NC-ND \\ https://doi.org/10.30820/1664-9583-2019-1-23
}

\begin{abstract}
Zusammenfassung: Die Psychoanalyse konnte in islamischen Gesellschaften in der Vergangenheit als Behandlungsmethode nur marginal Fuss fassen. Begründet wird diese Randstellung damit, dass Islam und Psychoanalyse im Widerstreit zu einander ständen. Muslim*innen würden aufgrund ihrer Religion im Kontext einer nach islamischen Normativitäten strukturierten Gesellschaft vom psychoanalytischen Behandlungsansatz nicht profitieren können. Für Muslim*innen der Schweiz sieht die Ausgangslage jedoch anders aus. Beispielhaft wird anhand von drei Behandlungsvignetten von muslimischen Migrant*innen dargelegt, dass sie von einer psychoanalytischen Psychotherapie profitieren können. Der Artikel beschreibt die Psychotherapieverläufe samt der sie einrahmenden Wechselwirkungen von psychopathologischen Beschwerden, innerpsychischen Konflikten und äusseren Lebenslagen im Kontext der jeweiligen individuellen muslimischen Religiosität.
\end{abstract}

Schlüsselwörter: Psychoanalyse, psychoanalytische Psychotherapie, Muslim*innen, Islam, Migration, psychoanalytische Fallvignetten, Schweiz

\begin{abstract}
«Wenn Sie zum Beispiel die Muslime verstehen wollen, die sich jeden Freitag in der Moschee in Ihrem Stadtteil zum Gebet versammeln, dann sollten Sie nicht nach einem perfekten System von Werten suchen, die allen Muslimen lieb und teuer sind. Suchen Sie lieber nach Konflikten und Widersprüchen, mit denen sich die Muslime dauernd herumschlagen und die niemand lösen kann. Eine Frage, auf die kein Muslim eine Antwort hat, ist ein Schlüssel zum Verständnis seiner Kultur.»
\end{abstract}

Harari (2013, S. 204)

In meiner psychoanalytischen Praxis als Psychotherapeutin arbeite ich mit etwa 24 Patient*innen pro Woche zusammen. Aufgrund meines Hintergrunds als feldforschende Soziologin im arabischen Raum und meinen Interessen im Bereich der Ethnopsychoanalyse haben gut $50 \%$ meiner Patient*innen einen Migrationshintergrund. Rund die Hälfte davon sind Muslim*innen: zum Beispiel die junge syrische Mutter, die mit ihrer Ankunft als Geflüchtete in der Schweiz ihr Kopftuch ablegte und ihre Verwandtschaft damit irritierte; die gestandene syrische Berufsfrau, die traurig ist, dass sie mit Schleier in ihrem angestammten Beruf nur schwer hier wird arbeiten können; der junge iranische Asylbewerber, dessen Familie in seiner Heimat den Kontakt zu ihm abbrach, da er auf einem Facebook-Foto mit einer goldenen Rapper-Kreuzkette abgelichtet wurde, oder der bald 30-jährige Kosovare, der nach einer Kindheit mit viel häuslicher Gewalt, als junger Erwachsener psychisch zu angeschlagen ist, um arbeiten zu können und der sich in die Haut tätowieren liess «only god knows about me». Oder schliesslich die junge Jordanierin, die aufgrund körperlicher Beschwerden und Einschränkungen gerne sterben würde, aber als Muslimin keinen Freitod begehen sollte und sich in ihrem familiären Umfeld mit ihren Todeswünschen unverstanden und einsam fühlt. In den Psychotherapien mit diesen Menschen spielt die Religionszugehörigkeit in ganz unterschiedlicher Weise eine Rolle. Es gibt dabei nur selten Gemeinsamkeiten mit den stereotypen Problematiken, die der öffentliche Diskurs prägt, wie etwa die Frage des Verweigerns des Händeschüttelns, der Radikalisierung von jungen Männern oder schliesslich der Unterdrückung als muslimische Frau.

Im Nachfolgenden möchte ich den Fächer aufspannen rund um die muslimische Religionszugehörigkeit von Patient*innen. Mich leitet dabei die Frage, welche Wechselbeziehungen zwischen psychopathologischen Beschwerden, innerpsychischen Konflikten und äusseren Lebenslagen im Kontext muslimischer Religiosität eines Individuums hier in der Schweiz in die psychotherapeutischen Praxen hineingetragen werden können und die psychotherapeutische Arbeit einrahmen und beeinflussen. Die muslimische Zugehörigkeit betrachte ich als einen Teilbereich der Kultur der Patient*innen. Dieser Bereich zeichnet sich durch den persönlichen Glauben, das Verhältnis zu Gott, zur islamischen Glaubensgemeinschaft und zur Relevanz der Religion im alltäglichen Leben aus. 
Diese Dimensionen fasse ich unter dem Begriff der muslimischen Religiosität zusammen. Damit eine Psychotherapie mit muslimischen Patient*innen gelingen kann, ist es wichtig, dass sich Psychotherapeut"in und Patient"in, die sich aufgrund ihrer unterschiedlichen soziokulturellen Zugehörigkeit und ihres kulturspezifischen Wissens, welches das Bewusstsein und das Unbewusste konfiguriert, vertrauter oder fremder vorkommen, begegnen können und ein gemeinsamer Prozess in Gang kommt: ein modernes Heilungsritual (vgl. Nathan, 1994).

Das Generieren von Einsicht ist im psychotherapeutischen Prozess für psychoanalytische Psychotherapeut*innen handlungsleitend: von unbewusst zu mehr Bewusstsein. Dabei handelt es sich um die Gestaltung und Förderung von innerpsychischen Lern- und Veränderungsprozessen, die bewusst und unbewusst ablaufen. Die islamische Grunddoktrin, in deren Zentrum Gott, dessen Allmacht und seine unsichtbar leitende Hand stehen, kann solche Prozesse beeinflussen. Für die psychotherapeutische Arbeit kann es herausfordernd sein, leidende Menschen zu begleiten, in deren Leben der Glaube einen höheren Stellen- und Welterklärungswert hat als der psychotherapeutische "Gott» der Wissenschaftlichkeit, Vernunft und Logik. So schreibt Osseiran, eine libanesische Psychoanalytikerin, über ihre psychoanalytische Tätigkeit, dass ihr Patient"innen Ereignisse oder Erfahrungen häufig als Ergebnis von etwas, was Gott gewollt habe, darstellen. Oder dass sie die Fortsetzung der Psychoanalyse davon abhängig machen, «so Gott will». Sie hält angesichts dieser islamisch-fatalistischen Mentalität gewisser muslimischen Patient*innen fest: «In diesem Fall, wer ist für die Behandlung verantwortlich? [...] Angesichts dieses anderen 'Gottes` fühlte ich mich an einem Punkt in meiner Praxis hilflos. Was konnte ich tun, wie sollte ich reagieren?» (Osseiran, 2010, S. 98; Übers. d. A.). Die islamische Normativität kann sich aber auch in ganz anderer Gestalt zeigen. Die Sozialisation zum/zur Muslim*in ist nicht ein passiver Prozess, der ohne eigenes Zutun passiert und zum immer gleichen Ausgangspunkt einer Art gottergebenen Lebenshaltung und -führung führt, wie obenstehendes Beispiel suggerieren könnte. Die durch Sozialisation erfolgten Ausformungen von muslimischer Identität und Religiosität sind höchst individuell. Sie erfolgen über Interaktionen im Kontext von interpretativen Mentalisierungsprozessen und aktivem sozialen Handeln, die unter dem Begriff der Agency zusammengefasst werden können (vgl. Allenbach, 2019, S. 3f.).

Für die psychotherapeutisch-psychoanalytische Arbeit müssen somit laut Hamid (2008, S. 3f.) zwei Fallen umgangen werden: Die erste sei die Annahme, dass der Islam eine gewichtige Dimension im Leben von allen Muslim*innen ist, die zweite bestände darin, von einer monolithischen Annäherung zur muslimischen Psyche auszugehen. Zweifelsohne sei die islamische Tradition ein wichtiger Einflussfaktor. Ein zwanghafter Persönlichkeitstyp könne durch die stetige Bezugnahme auf Schriften der islamischen Jurisprudenz eine Stütze für die Organisation seines alltäglichen Lebens erfahren. Eine gesunde muslimische Person mag auf resiliente Weise ein traumatisches Lebensereignis als Gottes Wille verarbeiten, ohne dass danach grössere innerpsychische Konflikte in eine Abwärtsspirale führen müssen. Kurzum, die Beziehung zur und die Prägung durch die islamische Tradition sei höchst individuell, ebenso wie deren kontinuierliche Rekonstruktion und Weiterentwicklung. Der Ausweg aus diesen Fallen besteht somit darin, die Einmaligkeit der muslimischen Patient"innen im psychotherapeutischen Prozess zu erfassen. Darauf weist auch Saller (2003, S. 428), die sich damit auseinander gesetzt hat, wie psychoanalytische Psychotherapien mit türkischen Frauen gelingen können, in einem Fallbeispiel hin. Eine Psychotherapie könne dadurch beeinträchtigt werden, wenn Patient" innen nicht in ihrer Individualität, sondern als «Angehörige von Kultur X» wahrgenommen werden. Dies könne verletzen oder Wut auslösen.

Ich steige in die umrissene Thematik mit einigen soziologischen Anmerkungen zur muslimischen Bevölkerung in der Schweiz ein. Ich spinne den Faden weiter, in dem ich einen kurzen Überblick über die Eckpfeiler des Islam als Religion und soziokulturelles System gebe. Ich skizziere im Anschluss die Geschichte und die Positionen des Verhältnisses von Psychoanalyse bzw. psychoanalytischer Psychotherapie und muslimischer Religiosität. Anhand von drei Fallbeispielen zeige ich dann auf, in welcher Form die muslimische Zugehörigkeit von Patient"innen im therapeutischen Prozess zum Thema werden kann. Für die Darstellung der Fallvignetten stütze ich mich auf die Methodik der dichten Beschreibung (vgl. Geertz, 1983). Das Datenmaterial hierfür bilden die von mir relativ umfassend geführten Krankengeschichten. Ich habe mir erlaubt, soziodemografische Eckdaten abzuändern, um die Anonymität meiner Patient*innen zu schützen. Dies verhindert jedoch nicht, dass aufgrund der Fallbeispiele sich zeigende idealtypische Problematiken dargestellt werden können.

\section{Die muslimische Bevölkerung der Schweiz}

In den meisten psychotherapeutischen Praxen wird der Anteil muslimischer Patient*innen gering sein, schliesslich beträgt ihr Anteil an der Schweizer Wohnbevölkerung lediglich 5,1\%, insgesamt rund 360000 Muslim*innen (SZIG, 2018): ein Bruchteil der weltweit geschätzten 1,5 Milliarden (Hamid, 2008, S. 3). ${ }^{1}$ Gemäss Schätzungen sind $10-15 \%$ der Muslim*innen der Schweiz praktizierend. Jede dritte muslimische Person ist im Besitz des Schweizer Passes (Herter et al., 2015) - in der Romandie scheint der Anteil etwas höher zu sein (Allenbach \& Sökefeld, 2010, S. 14).

Die Anzahl von Konvertit*innen weg vom Islam ist mit rund 5\% relativ gering (Bundesamt für Statistik, 2014, URL). ${ }^{2}$ Häufiger Anlass für das Konvertieren zum

1 Die meisten Muslim*innen leben in Südostasien. Insgesamt gibt es 50 islamische Staaten (Behloul, 2018).

2 Zum Vergleich: Im Schnitt wechselt jede fünfte Person in der 
Islam ist das Eingehen einer Ehe mit einem/r Muslim*in. Eine spezielle, kleine Untergruppe der Schweizer IslamKonvertit*innen bildet diejenige, die sich äusserlich an der Lebensweise der frühen Muslim*innen - mit Henna gefärbtem Vollbart oder Burka-Dresscode - orientiert und in den Medien grosse Aufmerksamkeit bekommt. Hafner Al Jabaji (2011) bezeichnet diese als neohelvetische oder pseudoarabo Idealmuslim"innen.

Das grösste Anwachsen der muslimischen Bevölkerung geht auf den Balkankrieg zurück, der in den 1990er Jahren zu einer deutlichen Erhöhung führte (Allenbach \& Sökefeld, 2010, S. 13). Ein Ausrufezeichen verdient hierbei die Tatsache, dass $90 \%$ der Muslim*innen der Schweiz aus Europa selbst - dem ehemaligen Jugoslawien - oder der politisch zu Europa zählenden Türkei stammen (Allenbach, 2019, S. 1). Der Bürgerkrieg in Syrien hat aktuell zu einer Aufnahme von weiteren rund 13000 Menschen mit muslimischer Religionszugehörigkeit geführt. ${ }^{3}$ Viele davon wurden durch Kriegs- und Fluchterfahrungen traumatisiert und sind aktuell auf psychotherapeutische Behandlungen angewiesen, ohne dass sie der deutschen Sprache mächtig sind. Zuvor waren es Muslim*innen aus dem Libanon und Irak, die infolge des (Bürger-)Kriegs Aufnahme in der Schweiz fanden. Anders als in den USA oder in Kanada gehören Schweizer Muslim*innen im Schnitt zu den ärmeren Schichten mit einem geringeren Bildungsniveau. Dies schränkt ihre beruflichen Möglichkeiten und, damit verbunden, ihre Einkommenschancen ein (Allenbach \& Sökefeld, 2010, S. 14).

Alles in allem ist die muslimische Landschaft der Schweizer Bevölkerung mindestens ebenso heterogen, wenn nicht sogar bunter, als die christliche. Sie zeichnet sich durch eine Aufsplitterung in viele kleine religiöse wie säkulare Gruppen und Organisationen aus, die kaum über politischen Einfluss verfügen. Ihre Glaubensvorstellungen und religiösen Praktiken können sich sehr unterscheiden (vgl. Allenbach \& Sökelfeld, 2010; D’Amato, 2015). Die dominante muslimische Gruppe in allen Landesteilen sind Sunnit*innen, die nach nationaler Herkunft und gemeinsamer Sprache - wie die meisten muslimischen Gemeinschaften - in Untergruppierungen organisiert sind. Zahlenmässig stammen $56 \%$ der Muslim*innen aus dem ehemaligen Jugoslawien (Kosovo-Albanien und Bosnien). Weitere 20\% haben türkische Wurzeln, 4\% kamen aus den Maghreb-Staaten und 3\% aus dem Libanon. Eine äusserst heterogene Gruppe von $15 \%$ bilden Muslim*innen aus Schwarzafrika und Asien. Neben der sunnitischen Mehrheit (75\%) leben in der Schweiz ca. 12000 Schiit*innen, vorwiegend iranischer Herkunft. Hinzu kommen türkische Alevit*innen (10-15\%) (Behloul, 2018).

Wie Allenbach und Sökefeld (2010, S. 12ff.) beschreiben, sind erste islamische Einflüsse in der Schweiz bereits für das 10. Jahrhundert nachzuweisen. Eine deutliche Sichtbarkeit erreichte die islamische Präsenz

Schweiz im Lauf des Lebens ihre religiöse Ausrichtung (ebd., S. 13). 3 Zwischen April 2011 bis Oktober 2016 sind 13713 Asylgesuche von Syrer"innen registriert worden (Amnesty International, 2017). aber erst - um ein Beispiel zu nennen - 1963 mit der Eröffnung der Mahmud-Moschee in Zürich. 1970 umfasste die muslimische Religionsgemeinschaft erst $0,26 \%$ der Schweizer Bevölkerung. Während sich in der Deutschschweiz vor allem Türkei- und Balkan-stämmige Muslim*innen niederliessen, sind es im frankophonen Teil Muslim*innen aus Nordafrika und den arabischen Ländern. In allen Landesteilen leben sie vorwiegend in den städtischen Ballungszentren. Muslim*innen sind somit oft Migrant*innen, wobei die Migration nur selten in missionarischer Absicht erfolgte. Hauptmotive waren und sind Arbeit und Flucht.

\section{Islam als europäischer Fremdkörper}

Die Beziehung zwischen islamischer Religion und Schweiz ist ein brisantes Thema, dem heutzutage auf der politischen Bühne viel Platz eingeräumt wird. Dabei vermischen sich die Diskurse über Muslim*innen und ihre Religion mit den Diskursen über Einwanderung, Heimat, Überfremdung, Extremismus und Terrorismus (vgl. Allenbach \& Sökefeld, 2010, S. 9). Gemäss Behloul (2011, S. 17) hat der heute zentrale Integrationsdiskurs über fremde Muslim*innen den früheren allgemeineren über Ausländer*innen abgelöst. Eine gewichtige Herausforderung für Muslim*innen in West- und Mitteleuropa besteht dabei darin, dass der Islam als etwas Fremdes betrachtet wird, als soziokultureller Fremdkörper, der nicht in das westliche Gesellschaftssystem hineinpasst. Der Islam kann in Europa sein, gehört aber nicht richtig zu Europa. Hand in Hand mit der Konzeptualisierung des Islam als soziokulturellen Fremdkörper geht dabei auch eine Konzeptualisierung von Europa durch Europäer*innen einher, die den Islam, da er als problematisch wahrgenommen wird, ausschliesst (Asad, 2003; zit. n. Allenbach, 2010, S. 297). So wurde beispielsweise im Dezember 2018 die Ablehnung der Anerkennung des Islam als weitere Religionsgemeinschaft im Kanton Bern in den Medien damit kommentiert, dass dies richtig sei, da «die islamische Gemeinschaft mit ihrem umfassenden Deutungsanspruch", anders etwa als die jüdische Gemeinschaft in den USA, noch kein "genügendes Bewusstsein» entwickelt habe für die eigene Identität in einer demokratischen westlichen Gesellschaft (Dütschler, 2018, S. 17). Dass diese Fremdkörperkonstruktion auch im Kleinen unwillentlich und subtil passiert, realisierte ich beim Schreiben dieses Artikels: Mein Arbeitstitel lautete «Psychoanalytische Psychotherapie mit Muslim*innen in der Schweiz", nicht etwa "Muslim*innen der Schweiz». Schweiz und Islam auf Anhieb zusammen zu denken, scheint anspruchsvoll.

Historisch kann daran erinnert werden, dass der Islam nicht nur vor unseren Toren stand, sondern beispielsweise in Spanien rund 400 Jahre von Bedeutung war. Auch heute ist er im Balkan Teil von Europa. ${ }^{4}$ Anders

4 An die Besonderheit der Muslim*innen des Balkans sei hier noch kurz erinnert: Viele von ihnen wurden erst im 17. Jahrhundert 
als andere Religionen oder von einer Religion gespiesene Praktiken eckt der Islam im westeuropäischen Selbstverständnis jedoch an. Während die sich zu Fitnesszwecken verrenkenden Yogakörper Bewunderung hervorrufen, die vom Buddhismus inspirierten achtsamkeitsorientierten Meditations- und Therapieverfahren leicht übernommen werden, befremden muslimische Rituale. Ramadan-Fasten ist nicht «in», der Ruf des Muezzins ${ }^{5}$ darf nicht öffentlich gehört werden.

Eine antiislamische Haltung hat sich in den europäischen Einwanderungsländern erst in den letzten 30 Jahren zunehmend verbreiten können. Im Kern wird angezweifelt, dass muslimische Eingewanderte respektable, gleichartige und gute Mitbürger*innen sein könnten (vgl. Giani, 2016; Allenbach, 2019). Auf muslimischer Seite besteht demgegenüber die Tendenz, sich aufgrund dieser antiislamischen Haltung diskriminiert zu fühlen. Eine arabisch sprechende Dolmetscherin, mit der ich für dolmetschergestützte Psychotherapien zusammenarbeite, berichtete mir zum Beispiel, dass es ihr reflexhaft immer wieder passiere, dass sie überprüfe, ob ein gewisses negatives Verhalten eines Gegenübers wohl damit zu tun habe, dass sie Muslimin sei; und dies, obwohl sie eine Schweizer Mutter habe und fliessend Schweizerdeutsch spreche.

Die sich entwickelnden antiislamischen Positionen können teilweise an der quantitativ grösseren Präsenz von Muslim*innen festgemacht werden. So waren ja auch die italienischen Gastarbeiter*innen Vorbehalten und Abwehr ausgesetzt. Dennoch ist es auffallend, dass Anti-Haltungen zwar bei gesellschaftlichen Veränderungen auftreten (früher gegen die Ex-Jugoslaw*innen oder gegen die Tamil*innen), jedoch im unterschiedlichen Mass an Terrain gewinnen. Im Moment scheint es eine offene Frage, ob die Konzeptualisierung des Islam als Fremdkörper bestehen bleiben wird oder ob sich diese Konstruktionen durch vermehrte Integrationsbestrebungen aufweichen werden. Es gibt Annahmen, dass in 20 Jahren Muslim*innen zur Schweiz gehören werden, ohne mehr die Irritationen und islamophoben Reaktionen von heute wachzurufen. Zurzeit werden von den Universitäten Fribourg und Bern erste Fortbildungsgänge für Imam*innen angeboten (vgl. Meier, 2018), die möglicherweise die Abgrenzungskonstruktionen zwischen Schweiz und Islam allmählich verändern können. Noch ist dies aber Zukunftsmusik für die Schweizer Gesellschaft. Das Minarettverbot zeugt davon.

Es ist hier nicht der Platz, um in aller Tiefe die Dynamiken und Ursachen dieser soziokulturellen Fremdkörperkonstruktion $\mathrm{zu}$ analysieren. Festgehalten werden kann jedoch, dass damit verbunden gesellschaftliche

gewaltsam islamisiert. Sie wurden in den 1980er Jahren "wiederentdeckt», wobei drei Diskurse auszumachen sind: als auszustossender Fremdkörper im Sinne des Kampfes der Zivilisationen, als oberflächliche, potenziell wieder zu Christ*innen zu bekehrende Muslim*innen und schliesslich als besondere, weil eben europäische Muslim*innen (Clayer, 2003, S. 308; zit. n. Allenbach \& Sökefeld, 2010, S. 15).

5 In den USA scheint es erste weibliche Muezzins zu geben (vgl. Sheena, 2018).
Imaginationen über den Islam als problematisch und unpassend einhergehen, beispielhaft verkörpert durch die in schwarze Burkas eingehüllten saudischen Touristinnen in Interlaken, die durch das kollektive Bewusstsein der postmodernen europäischen Gesellschaften geistern. Der Islam wird in den Vorstellungen von durchschnittlichen westlichen Bürger*innen von einem Unterton des Fanatischen und Gefährlichen umspielt (vgl. Akthar, 2008, S. xiv). Dies führt wohl auch mit dazu, dass die Schweizer Bevölkerung, so eine repräsentative Umfrage eines Medienunternehmens, die Anzahl Schweizer Muslim*innen deutlich überschätzt. Die Befragten schätzten deren Anteil an der Schweizer Wohnbevölkerung auf 17,2\% (Birrer, 2017). Muslim*innen sind durch diese Diskurse in dem Sinn belastet, dass sie eine eigene Haltung dazu finden müssen: Sind wir effektiv die muslimischen "Anderen» und wann spielt dies für uns auf welche Weise eine Rolle?

\section{Islamische Normativität - eine Annäherung}

Mir scheint es wichtig, dem Verständnis eines problematischen Islam ein anderes Verständnis des Islam gegenüber zu stellen, das muslimischen Selbstrepräsentanzen näherkommt als die der öffentlichen Mainstream-Diskurse. Versetzen wir uns in das 7. Jahrhundert, als Mohammed durch die Erscheinung des Erzengels Gabriel Gottes Wort vernahm. Dies passierte erstmals im Jahr 610 und dauerte bis 632, dem Jahr seines Todes. Die durch den Propheten Mohammed empfangenen Worte Gottes, eines als perfekt und ausgeglichen geltenden Mannes, waren für die damalige Zeit ein radikaler Durchbruch hin zu einem humaneren, fürsorglicheren und gleichwertigen Miteinander von Kindern, Frauen und Männern, Armen und Kranken, eingebettet in den Anspruch eines wirtschaftlich fairen Handels: Das lebendige Vergraben von ungewollten weiblichen Säuglingen wurde verboten, ebenso wie die Misshandlung von Waisen und Armen. Frauen bekamen Erbrechte zugesprochen, sie wurden als vor Gottes Augen spirituell ebenbürtige betrachtet. Die Autorität von Stämmen wurde eingeschränkt, sie wurden aufgefordert, sich der wahren Religion des Islam zu beugen. Mohammed begann durch seine Botschaften Anhänger*innen zu gewinnen, war jedoch ohne politische Schutzmacht. Es begann eine Zeit der sozialen Erniedrigung, ökonomischen Sanktionen und physischer Gewalt für die frühen Muslim*innen. Diesen Schwierigkeiten begegneten die Konvertit*innen mit der genannten tawakkul, dem resilienten und beharrlichen Glauben in schwierigsten Lebensbedingungen; sie wurden so zu einem instruktiven Beispiel für viele Muslim*innen bis heute. 13 Jahre nach dem ersten Vernehmen von Gottes Wort floh Mohammed mit mehreren tausend Anhänger*innen von Mekka nach Medina. Dieser Zeitpunkt markiert die islamische Zeitrechnung als Jahr 0. Es ist der Start der muslimischen Gemeinschaft, der ummah: In der Fremde, getrennt von Familien, Stämmen und früherem Leben entwickelte sich diese Gemeinschaft vom marginalisierten und unterdrücken Kult zu einer wachsenden religiösen 
und politischen Gemeinschaft, innerhalb derer sich alle als Brüder und Schwestern betrachteten. Diese MedinaGemeinschaft diente und dient noch heute als idealisiertes Modell einer islamischen Gesellschaftsordnung (Hamid, 2008, S. 7ff.).

Islam geht etymologisch auf das arabische Verb salama zurück. Neben seinen Wurzeln im Wort Frieden, bedeutet es als Verb «sich zu beugen». Ein*e Angehörige*r des Islam unterwirft sich somit Gottes Willen. Eines der zentralen religiösen Konzepte des Islam ist dabei, dass die Menschheit in der Schuld des allmächtigen allwissenden Gottes, der das Universum schuf, steht. Anders als der alttestamentarische Gott vergibt der islamische Gott den bereuenden Adam und Eva. Gleichzeitig liegt es in der menschlichen Natur, die Schuldigkeit gegenüber Gott zu vergessen. Durch die täglichen religiösen Rituale werden die Gläubigen daran erinnert, dass sie achtsam sein müssen angesichts ihrer weltlichen Handlungen und gewahr sein müssen ihrer moralischen Verantwortung, Gott und seine Schöpfung zu ehren und die affektive Beziehung zu Gott zu pflegen (ebd., S. 6).

Das Selbstverständnis von Muslim*innen ist bis in unsere Zeit davon geprägt, dass ihre religiöse Geschichte festgehalten und niedergeschrieben wurde, während sie sich ereignete. Sie wurde auswendig gelernt und weitergegeben. Das Empfangene, in 114 Suren geordnet, galt als direktes Wort Gottes, das nicht korrigiert und mit den enthaltenen Widersprüchen angenommen werden soll. Die Geschichten und Erzählungen wurden dabei nicht als Gleichnisse betrachtet, sondern als wirkliche Erfahrungen von wirklichen Menschen, die danach trachten, in einer Gemeinschaft zusammen zu leben (Ansary, 2010, S. 20).

Zurück in die Schweiz: In einer Gesellschaft, in der die religiöse Macht von der politischen und verwaltenden Macht abgetrennt ist, stellt sich die Frage, was von Gott in unserem postmodernen Gesellschaftssystem unter einer Staatsmacht übrig bleibt. Religionen haben sich hier, um es mit Vincent (2016, S. 15; Übers. d.A.) zu sagen, folgendem Prinzip unterzuordnen: «Gott darf eines jeden Angelegenheit bleiben, ohne dadurch eine Angelegenheit aller zu sein.»

Was bedeutet dies für Schweizer Muslim*innen? Der Islam umfasst, wie oben stehend geschildert, mehr als ein Glaubenssystem. Er ist ein eigenes Universum im Sinne eines sozioökonomischen Gesellschaftsprojektes mit Konzepten zur Ordnung von Politik und Wirtschaft sowie einem vollständigen Zivil- und Strafrecht. Dieses betrifft auch die Geschlechterbeziehungen - so etwa die Trennung der Geschlechter im öffentlichen Raum zur Regulierung ihrer Sexualität, die im privaten Raum relativ offen und lustvoll sein kann. In religiöser Hinsicht weist er hingegen viele Ähnlichkeiten mit der christlichen und jüdischen Religion auf; die Vorstellungen zu Ethik, Moral, Gott, dem Kosmos und dem Jenseits ähneln einander (vgl. Ansary, 2010, S. 347).

Schweizer Muslim*innen darauf zu reduzieren, dass sie dieses islamische Gesellschaftsprojekt vorantreiben wollen, verfehlt die aktuelle Situation der muslimischen Bevölkerung. Angesichts ihrer Heterogenität, ihrer Mi- grationserfahrungen und ihrer tendenziellen Marginalisierung ist das ihnen Gemeinsame, dass sie hier in Europa gleichsam in ihrer religiösen Identität auf einer Sandbank gelandet sind, die unterspült zu werden droht durch die Strömungen eines einseitig verzerrten Islam. Sie erkennen hierbei sich und ihr Verständnis der islamischen Tradition, mit den Gemeinsamkeiten und der Anschlussfähigkeit an die christliche Normativität, in der negativen Wertigkeit nicht wieder. Aktuelle europäische Forschungen erhellen, dass bei Muslim*innen in ihrer Suche nach einer Lebensweise innerhalb einer islamischen Normativität auf dem Boden der eigenen Erfahrungen immer auch die Auseinandersetzungen mit der nationalen Normativität miteinfliesst, sei es die der Schweiz, von Frankreich oder Deutschland. In Bezug auf die Geschlechterfrage gibt es interessanterweise Hinweise darauf, dass gerade Frauen hierbei in emanzipativer Weise sich ein eigenes Verständnis des Islam anzueignen versuchen. Diese Forschungen zeichnen ein Bild von Muslim*innen als souveräne Subjekte, die selbstbestimmt über ihren Glauben verfügen - auch wenn sie einer exklusivistischen islamischen Lebensführung zugewandt sind. Sie orientierten sich dabei an weiblichen Vorbildern, wie beispielsweise den Ehefrauen des Propheten Mohammed, die nicht dem Bild einer unterdrückten Frau entsprechen (Bleisch, 2016, S. 17ff.).

\section{Psychoanalytische Psychotherapie für muslimische Patient*innen?}

Die Frage des Verhältnisses zwischen muslimischer Religiosität und psychoanalytischen Psychotherapieprozessen wird in zwei wissenschaftlichen Feldern bearbeitet: einerseits in der transkulturellen Psychiatrie, die sich mit der Frage des Einflusses von Kultur in Behandlungssettings- und verfahren auseinandersetzt und so auch immer den Religionsaspekt miteinbezieht, andererseits durch die geschichtliche und soziologische Aufarbeitung der Entwicklung der Psychoanalyse. Hier ist der Ausgangspunkt der Debatten die Tatsache, dass sich die Psychoanalyse sehr ungleich über den Globus ausgebreitet hat. Islamische Gesellschaften blieben gleichsam von der Psychoanalyse ausgespart. Ausser in Ägypten gab es in keinem islamisch geprägten Land eine massgebliche Aneignung der Psychoanalyse bis gegen Ende des 20. Jahrhunderts. Für die geringere Etablierung der Psychoanalyse in islamischen Gesellschaften fanden sich Erklärungen wie die, dass Individuen zu stark in Gemeinschaften eingebunden sind, dass das islamisch geprägte gesellschaftliche Leben stärker reglementiert und normiert ist oder schliesslich auch der muslimische Glaube selbst, der Unglück als Gottesprüfung oder gottgewolltes Schicksal betrachtet (vgl. El Khayat, 1994; Hammad, 2004). Studien über die arabo-muslimische Welt betrachteten als Hindernisse für die Verbreitung der Psychoanalyse die das Denken formende Sprache des Arabischen, der Ursprungssprache des Koran, in der es beispielsweise das Wort sein nur in der Vergangenheit gibt (Melman, 2004, S. 4). Auch der Begriff des Ichs existiert nicht in der gleichen subjektbe- 
zogenen Färbung. Nicht zuletzt wurde auch angeführt, dass in politisch repressiven Ländern, das freie Denken eingeschränkt ist, und so auch die Psychoanalyse: Wie soll hier jemand dazu eingeladen werden, ohne Selbstzensur auf assoziative Weise alles zu sagen, was in den Sinn kommt (vgl. Houballah, 2004, S. 20)?

Die frühe Auseinandersetzung mit der Psychoanalyse in Ägypten zeigt jedoch, dass lokale Konstellationen in diese Erklärungsversuche miteinzubeziehen sind und die Problematisierung der Psychoanalyse immer auch einen historischen Kontext hat. Die Psychoanalyse erlebte in Ägypten in den 1930er bis 60er Jahren eine Blütezeit oder zumindest eine lebendige, umtriebige Phase. Sie wurde rezipiert, praktiziert und durch Übersetzungen und Publikationen einem breiteren Publikum zugänglich gemacht. Ägyptische Wissenschaftler*innen beleuchteten psychoanalytische Modelle und versuchten diese mit klassischen islamischen Konzepten in Verbindung zu setzen. Sie glichen einzelne Theoriebausteine miteinander $\mathrm{ab}$ und schufen neue hybride Ansätze zu Fragen von Persönlichkeit und Individualität (vgl. El Shakry, 2014). Eine der Hauptprotagonisten war Mustapha Ziwer, der in Frankreich seine psychoanalytische Ausbildung absolvierte und später an der alexandrinischen Universität Farouk lehrte. Es war selbstverständlich in dieser Phase des späten Kolonialismus, sich als wissenschaftlich interessierte Subjekte und interpretierende Handelnde mit der fremden Psychoanalyse zu beschäftigen, während gleichzeitig die britische Besatzung bekämpft wurde (Kader, 2004, S. 64). Das skeptische Bewusstsein dafür, dass die Auseinandersetzung mit der Psychoanalyse für Behandelnde oder Erkrankte problematisch sein könnte oder dass sich die islamische Normativität an der Psychoanalyse aufreibt, fehlte.

Je mehr die Psychoanalyse in anderen nicht-westlichen Gesellschaften durch Professionalisierungsprozesse Einzug hielt, desto intensiver wurde aufgrund der gemachten Erfahrungen über Praktikabilität und Angemessenheit des Verfahrens nachgedacht. Dazu trugen auch die aufkommenden postcolonial studies bei. Unter deren Ägide wurden Fragen zur Dominanz und Richtigkeit der westlichen Weltsicht gestellt, die zweifelsohne auch in den Konzepten der Psychoanalyse zu finden ist. Ist es richtig, Behandlungsansätze, die modernen westlichen Denktraditionen entspringen, in weniger individualistisch ausgerichteten Gesellschaften einzuführen? Der Psychoanalyse wurden Vorwürfe gemacht. Sie habe, so Kakar (1999, S. 13), im besten Fall «die Möglichkeit, dass andere Kulturen, mit ihren andersartigen Weltanschauungen, Familienstrukturen und Beziehungen einen eigenen Beitrag zur Entwicklung psychoanalytischer Prinzipien und Modelle leisten könnten, einfach ignoriert». Noch fragwürdiger seien die Intentionen von einigen Psychoanalytiker*innen gewesen, kulturspezifische Pathologien wie etwa orale Fixierung und nicht überwundene Abhängigkeiten zu entwerfen, ohne sich vertieft damit auseinanderzusetzen, wie etwa «Befriedigung», «Stimulation» oder «Frustration» eines Menschen in einem völlig anderen soziokulturellen Kontext qualitativ beurteilt werden kann. Vielfach hätten sich dabei kulturelle Wertigkeiten mit wissenschaftlichen Erklärungen vermischt (ebd., S. 15).

Auch die Wirksamkeit der Psychoanalyse wurde bezweifelt, da in islamische Gesellschaften statusbezogene Kommunikationshemmungen bestehen und Selbstenthüllungen sozial weniger akzeptiert sind. Eine weitere Ursache dafür, dass die psychoanalytische Methode in islamischen Gesellschaften auf unfruchtbaren Boden fiel, wurde darin gesehen, dass hier die Unterscheidung zwischen psychischem und physischem Wohlbefinden schwächer ausgeprägt sei. Generell wurde für Muslim*innen als geeigneter erachtet, nach konkreten Lösungen zu suchen statt nach tiefschürfenden Einsichten (Al-Issa, 2000, S. 345). Zudem zeigte sich, dass die psychoanalytischen Konzepte nicht in die religiös und wissenschaftlich akzeptierten Erfahrungswelten passten und Patient*innen dazu tendierten, in pseudoreligiösen Verfahren Zuflucht zu suchen - so beschrieben beispielsweise von Azhar \& Varma (2000, S. 176) für das islamische Malaysia. Al-Issa (2000, S. 342f.) urteilt, dass psychoanalytische Konzepte mit der basalen islamischen Doktrin inkompatibel seien. Der Koran würde ein gänzlich anderes Menschenbild vertreten.

Im Zuge der Professionalisierung des psychiatrischen Felds wurden daher andere Psychotherapieverfahren entwickelt, die erfolgversprechender schienen und den Bedürfnissen von muslimischen Patient*innen besser entsprachen - bis hin zu explizit islamischen Psychotherapien, die sich auf islamische Werte und damit verbundene Menschenbilder abstützen. Als vielversprechender wurden kognitiv-behavioristische Therapieansätze betrachtet, durch welche die Ideale und das Wertesystem von Patient*innen verändert werden könnten, sodass sie mit der muslimischen Religion in Einklang ständen (ebd.): «Die Ziele der Therapie im islamischen Kontext sind nicht Unabhängigkeit und Selbstverwirklichung, sondern Interdependenz und Anpassung an die Anforderungen der Gesellschaft» (ebd., S. 347; Übers. d.A.). Gerade Gruppenpsychotherapien, eingebettet in einen religiösen Kontext, hätten sich als nützlich und wirksam erwiesen (ebd.). Azhar \& Varma (2000, S. 176) setzen mehr auf supportive Techniken, mit dem Ziel «die Kraft der Patient*innen zu maximieren, ihre Abhängigkeit von den Therapeut*innen zu minimieren und ihnen zu helfen, so unabhängig wie möglich zu leben» (ebd.; Übers. d.A.). Dies würde auf der Basis der Aufrechterhaltung und Stärkung der Persönlichkeitsstrukturen und Abwehrmechanismen gelingen. Als weitere Eckpfeiler einer Psychotherapie, die mit einem islamischen Menschenbild in Einklang steht, gelten die Betonung emotionaler Zurückhaltung, das Erfüllen erwarteter sozialer Rollen und der Fokus auf Interdependenzen innerhalb von Gemeinschaften. Vom psychisch autonomen Innenleben eines Individuums mit seinen Motiven, Werten, Charakterzügen und Begehren wird Abkehr genommen. Priorität haben die Ziele der Gemeinschaften, zu denen eine Person zugehörig ist, auch wenn diese Ziele in Konflikt mit persönlichen Zielen ständen (Al-Issa, 2000, S. 319).

Diese Studienergebnisse und Überlegungen können nicht eins zu eins auf die in den Westen migrierten 
Muslim*innen übertragen werden. Die Reichweite der Psychoanalyse ist hierzulande grösser aufgrund der Strukturen des Schweizer Gesundheitssystems und hier vorherrschenden wissenschaftsbasierten Krankheitskonzepte. Für die Schweiz müsste man eher von einer tendenziellen Unterversorgung von psychisch kranken Muslim*innen sprechen aufgrund ihrer kulturspezifisch anderen Krankheitskonzepte: Von selbst begeben sie sich seltener in psychoanalytische Praxen. Tauchen sie jedoch auf dem Radar des Gesundheitssystems auf, kann es passieren, dass sie für eine Therapie überwiesen werden. Dies passiert tendenziell immer öfter, weil sich auch in der Schweiz die transkulturelle Psychiatrie vom «Orchideenfach» zum allgemeinen Therapiehandwerk gemausert hat und beansprucht, mit Muslim*innen auf kultursensible Weise arbeiten zu können (vgl. GraefCalliess \& Schouler-Ocak, 2017). Dieser Anspruch zeigt sich gleichfalls innerhalb der Psychoanalyse: Während die klassische Psychoanalyse eher im Rückzug begriffen ist, halten sich trotz Konkurrenz durch andere Therapieverfahren psychoanalytische Psychotherapien. Sie entwickeln sich in Richtung eines reflexiven und selbstkritischen psychoanalytischen Verfahrens weiter, in der transkulturelle und kultursensible Überlegungen ihren Platz haben. Der Aufschwung der intersubjektiven Psychoanalyse mag dabei eine Rolle gespielt haben. So wird verstärkt versucht, die soziokulturelle und religiöse Herkunft der Patient”innen zu berücksichtigen und psychoanalytische Prozesse auf diversifizierte Weise zu gestalten: Die Ansätze reichen von der Idee, die Tendenz von Patient*innen mit einem muslimischen Hintergrund, persönliche Konflikte ausserhalb von sich und der eigenen Lebensgeschichte zu verorten, als Widerstand zu deuten über die Empfehlung des aktiveren Einbringens als Psychoanalytiker* in - eine wärmere, empathischere Haltung - bis hin dazu, dass zuerst eine psychoedukative Vorarbeit geleistet werden müsse, wie beispielsweise Patient"innen in ihrer Selbstbeobachtung zu unterstützen sind (vgl. Kakar, 1999; Saller, 2003). Sie gehen aber auch von der Vorstellung aus, dass es universelle psychoanalytische Konzepte über das Entstehen, die Struktur, das Funktionieren und die Konflikthaftigkeit der menschlichen Psyche gibt, die ungeachtet der religiösen Zugehörigkeit eines Menschen von Bedeutung sind. Es existieren vergleichbare Kindheitserfahrungen innerhalb von Familienstrukturen quer durch alle religiösen Gemeinschaften. Khan (2008, S. 294) fasst zusammen, dass allen Menschen eine entwicklungspsychologische Infrastruktur zu eigen sei, die komponiert wird durch unbewusste, traumbasierte Kindheitserfahrungen. Diese persistiere im erwachsenen Menschen ungeachtet der in verschiedenen soziokulturellen Systemen durchlaufenen Sozialisationen.

Jenseits von all den Wissenschaftsdebatten besteht in der Praxis aber wohl die grösste Herausforderung darin, dass psychoanalytische Psychotherapeut"innen im Erstgespräch auf muslimische Patient"innen treffen, die mit einer vagen Hoffnung im Sprechzimmer sitzen, jedoch nur beschränkte bis gar keine Vorkenntnisse von einer psychoanalytischen Psychotherapie haben - Menschen somit, die zwar ein Bild einer ärztlichen Behandlung und der dadurch erhofften Besserung besitzen, jedoch keine eigentliche Vorstellung, wie und warum das assoziative Heilungsritual des sich um das Unbewusste drehenden Behandlungsansatzes hilfreich und nützlich sein könnte. Der Start einer psychoanalytischen Psychotherapie bedeutet somit öfter, Patient*innen dazu zu verführen, den Brückenschlag zu etwas Unbekannten zu machen. Ein gemeinsamer religiöser Hintergrund scheint dies zu erleichtern. In Deutschland gibt es beispielsweise ein Internetportal, das Psychotherapien mit muslimischen Psychotherapeut*innen vermittelt, darunter sind auch Psychoanalytiker*innen zu finden. Nichtsdestotrotz ziehen es einige Muslim*innen vor, zu nicht-muslimischen Therapierenden zu gehen, da sie sich von diesen mehr Offenheit versprechen. Sie fürchten, ihr Leben, Handeln und Fühlen vor dem Hintergrund eines «Masterplans» der islamischen Tradition und eines allwissenden Gottes im psychotherapeutischen Raum zu verhandeln; sie möchten vermutete Einschränkungen vermeiden. Des Weiteren bestehen auch Ängste, da sich die religiösen Gemeinschaften überschneiden könnten und es einfacher sein kann, sich gegenüber Psychotherapeut*innen zu öffnen, die der eigenen Alltagswelt fern sind.

\section{Ambivalente Lebenslagen und innerpsychische Konflikte von muslimischen Patient*innen - drei Fallvignetten}

Die nachfolgenden drei Fallbeispiele beschreiben, wie muslimische Patient*innen den Brückenschlag zur der in Mitteleuropa ersonnenen psychoanalytischen Behandlungsmethode gestalten. Ich gehe dabei auf ihre Konfliktlagen im Kontext ihrer muslimischen Religion näher ein. Sie verkörpern Beispiele für Auseinandersetzungsformen mit der «islamischen Normativität» (Bleisch, 2016, S. 15), die im psychotherapeutischen Prozess zum Thema werden können.

\section{Der Islam als Manual für das Leben}

Herr H. wurde von seiner Hausärztin für eine Psychotherapie überwiesen, da ihr die Schlaf- und Konzentrationsprobleme Sorgen machten, über die er sich beklagte. Sie erwog, ob er nicht aufgrund seiner Fluchtgeschichte an einer posttraumatischen Belastungsstörung leiden könne.

Herr H., ein sunnitischer Paschtune, stammt aus Afghanistan, wo er als Mathematiklehrer gearbeitet und parallel noch ein Zweitstudium begonnen hatte. Da sein Vater einen guten Posten bei der Regierung innegehabt hatte, war es ihm möglich gewesen, zwölf Jahre lang eine Privatschule zu besuchen. Er lebte bei Therapiebeginn seit anderthalb Jahren als Asylbewerber in der Schweiz.

Er sei in einem kleinen Dorf aufgewachsen, wie die meisten Afghanen, die noch zu 97\% von der Land- 
wirtschaft leben würden und arm seien: 25 Häuser, ein trockener Fluss, eine Moschee, die Erinnerung an ein Massaker der Russen, bei dem acht Verwandte gestorben seien; beinahe auch sein Vater. Das habe diesem fast die Sprache geraubt. Er sei im Krieg gross geworden, einem Krieg, der seit 40 Jahren bestehe.

Herr H. beschäftigten seine Schlaf- und Konzentrationsprobleme, da er vorhatte, ein Studium zu beginnen. Ihm war auf hartnäckige Gesuche hin gestattet worden, ein Hochschulstudium (mit Unterstützung von diversen Stiftungen) zu beginnen. Die Psychotherapie mit ihm dauerte ein knappes Jahr. Sie war von kurzen Unterbrechungen gekennzeichnet, da für ihn Studiums- und familiäre Verpflichtungen stets Vorrang hatten. Bei Beginn der Psychotherapie war er 25 Jahre alt.

Gleich im Erstgespräch erzählte er mir seine Fluchtgeschichte in aller Ausführlichkeit. Ausgangspunkt seiner Flucht war das unvermittelte Verschwinden seines Vaters gewesen, der politisch aktiv gewesen sei. Herr $\mathrm{H}$. startete einen vergeblichen Gang durch Behörden, Ämter, Regierungsstellen, Polizeiposten und Gefängnisse. Eine Woche später bekam er von seiner Mutter einen Anruf, dass sie gewarnt worden sei. Auch nach ihm würde gesucht, er müsse verschwinden. Daher tauchte er unter und gab seine Stelle auf.

Mit einer Schleppergruppe kam er schliesslich in die Schweiz. Es war eine traumatische Flucht. Zwei Mitglieder seiner Fluchtgruppe wurden bei einem Grenzübertritt erschossen. Die Reise über das Mittelmeer nach Griechenland wurde fast zu einer Reise in den Tod. Der Motor des aus Brettern und Schläuchen selbst angefertigten Schiffes fiel aus. In dieser Nacht auf dem Meer, als keine Hilfe kommen wollte und bereits Wasser in das Schiff einzudringen begann, muss Herr H. Todesängste ausgestanden haben: Er habe noch nie in seinem Leben so Angst gehabt. Erst in letzter Minute erklärte sich eines der vorbeifahrenden Frachtschiffe bereit, die Geflüchteten an Bord zu nehmen - mit Vermittlung von NGOs, die rund um die Uhr für Geflüchtete in Seenot erreichbar sind und deren Telefonnummer einer der Bootsinsassen auf seinem Handy gespeichert hatte.

Ich war berührt angesichts dieser Fluchtgeschichte, die er mir, mich kaum zu Wort kommen lassend, teils auf Deutsch, bei emotionalen Stellen ins Englische wechselnd, in cineastischen Bildern schilderte. Ich war aber auch irritiert über seine so runde Erzählweise, kam dann zum Schluss, dass er diese Fluchtgeschichte, die er in eine packende aufwühlende Erzählung umgewandelt hatte, so schrecklich die einzelnen Ereignisse auch gewesen waren, vermutlich verarbeitet hatte. Herr H. schien über Resilienzen zu verfügen. Was liess ihn nun nicht schlafen und raubte ihm die Konzentration?

Eine Konfliktlage kristallisierte sich rasch heraus: Er wollte alles perfekt machen. Dass er nach anderthalb Jahren fliessend Deutsch sprach und bereits als Übersetzer tätig war, schien diesen Anspruch an Perfektion zu bestätigen.

Zentrales Thema in der Therapie wurde dann eine Über-Ich-Problematik, die sich im Rahmen des angestreb- ten Studiums zuspitzte. Die Schweizer Hochschulwelt schien für den leistungsorientierten jungen Mann eine Überforderung zu sein. Es gelang ihm nicht, die geforderten Leistungen zu erbringen, obwohl er viel lernte. Zu sehr würde sich die Schweizer Hochschulwelt von der, die er gewohnt war, unterscheiden. Und doch: Er wollte mit dem Kopf durch die Wand und sein in Afghanistan angefangenes Studium in der Schweiz fortsetzen. Gleichzeitig trug er jedoch auch eine Verantwortung für seine Mutter und Schwestern. Da es ihm ein Anliegen war, ihnen Geld zu schicken, hatte das Übersetzen stets Vorrang. Ich denke, dass die Psychotherapie es ihm ermöglichte zu erkennen, dass der Abbruch seines Studiums keine Schmach und keine Schande sein müsste. Er konnte einen geordneten Rückzug von diesem Vorhaben antreten und dank eines ärztlichen Zeugnisses auch von den Studiengebühren befreit werden, da er sich nicht termingerecht exmatrikuliert hatte. Eine Rolle spielte dabei auch die Auseinandersetzung mit den Erinnerungen an seinen fürsorglichen, verantwortungsbewussten, aber auch strengen und fordernden Vater, von dessen angenommenen Erwartungen es ihm gelang, sich zu distanzieren.

Neben diesen Studienkonflikten gab es noch eine weitere Über-Ich-Problematik. ${ }^{6}$ Es ging dabei um eine Liebesbeziehung. Zuerst ging ich von widerstreitenden religiösen und kulturellen Vorstellungen aus, die ihm zu schaffen machten, wie sie beispielsweise in der zweiten Sitzung thematisiert wurden: Herr. H. skizzierte und positionierte sich mir gegenüber als ein sich den Grundsätzen des Islam verpflichtet fühlender Mann. Die Stunde begann damit, dass er schilderte, wie er seine Ankunft in der Schweiz erlebte: Es sei für ihn sehr unangenehm gewesen, hier in der Schweiz Franenarbeit verrichten zu müssen: putzen, waschen, kochen. Sein erstes «Ämtli» in der Asylunterkunft sei gewesen, die Toiletten zu putzen, das hätte er fast nicht fertiggebracht. Er empfinde dies als unrein. Und sehr entwürdigend für ihn als Mann. Zu Hause habe dies stets seine Mutter gemacht. Er habe sich nun aber etwas daran gewöhnt.

Nach der darauf folgenden Schilderung eines Albtraums - wir hatten seine Schlafprobleme angesprochen -, in dem er von einem monsterartigen Geist verfolgt wurde, der seinen Freund attackieren wollte und den er nicht richtig warnen konnte, kam er auf seine Beziehung zum Islam zu sprechen. Herr H. hatte wohl eine leichte Überraschung bei mir wahrgenommen, als er schilderte, wieso er voller Angst aufgrund des Albtraums aufgewacht sei: Es wäre furchtbar, wenn sein Freund von einem Geist befallen würde. Jedenfalls führte er aus: Er glaube an Geister, sie seien Kreaturen Gottes, schon der Koran habe diese beschrieben. Sie könnten, wenn sie verletzt würden, in die Körper der Menschen fahren. Die befallenen Menschen würden dann plötzlich mit anderer Stimme, neuen Kräften von diesen gesteuert werden. Er habe dies selbst gesehen. Sein Verbältnis zu Gott sei ihm wichtig,

6 Nicht vergessen werden soll, dass die Situation als Asylbewerber, über dessen Gesuch noch nicht entschieden ist, an und für sich sehr belastend ist. 
er glaube an den "day of judgment». Dass er erst nach allfälliger Bestrafung seiner Sünden in den Himmel käme. $\mathrm{O} b$ ich Computerhandbücher kennen würde? Im Grunde genommen sei der Koran solch ein Handbuch, ein Manual für die ganze Lebensgestaltung.

Rückblickend hätte ich wohl seine Schwierigkeiten rascher begriffen, hätte ich genauer auf unsere Beziehungsgestaltung und meine Gegenübertragungsreaktionen geachtet. Mich besorgte zu Beginn der Psychotherapie, dass trotz meiner Erklärungen über Methodik, Zweck und Verbindlichkeit einer Psychotherapie, das psychotherapeutische Arbeitsbündnis nicht zustande zu kommen schien. Er verschob die Sitzungen, wenn er die Möglichkeit zum Geld verdienen hatte, und vergass manchmal, mich darüber zu informieren. Wenn er da war, redete er gerne und ausführlich über seine Situation und all seine Schwierigkeiten. So wie er in diesen Stunden vor mir sass, die Beine locker überschlagen, entspannt wirkend, tauchten bei mir Bilder von Männern in englischen Clubs auf, denen ich Whiskey servierte. Ich denke, dass dieses mich störende Gegenübertragungsbild - sass ich doch einem Asylbewerber gegenüber, der sich in einer belastenden Situation befand im Zusammenhang mit seinen inneren Konflikten hätte verstanden werden können: Die Frage, wie sich Männer und Frauen hier in der Schweiz begegnen können und die Gefahr, dass dabei islamische Ordnungen verletzt werden.

Im Verlauf der Psychotherapie wurde dann allmählich deutlicher, worin diese Nebenthematik, die Herrn H. in Nöte brachte, bestand: Eine Mitarbeiterin der Asylunterkunft hatte ihm viel geholfen: Selina. Sie ergänzte mit dieser Arbeit ihr Einkommen zu ihrer eigentlichen Tätigkeit als Bildhauerin: Sie sei acht Jahre älter als er, sie habe sich in ihn verliebt, sich seinetwegen sogar von ibrem damaligen Partner getrennt. Sie seien jedoch äusserst unterschiedlich. In Afghanistan seien die Menschen viel ähnlicher gewesen, auch über Klassenschranken hinweg, er habe viele arme Freunde gehabt. Überhaupt, er habe dort enge Beziehungen zu Männern gehabt. Sein bester Freund habe am Flughafen lange geweint, als er abreiste. Er sei so lustig gewesen, habe ibn aus traurigen Stimmungen herausreissen können. Er vermisse seine Freunde mehr als seine Familie und Verwandten.

Herr H. ist in dieser Beziehung zu Selina sehr ambivalent. Ihre unterschiedlichen sozioökonomischen Verhältnisse, eine Frau, die ihm als Asylbewerber Dinge ermöglicht, die sogar so weit gehen würde, ihn zu heiraten, damit er hier bleiben könnte. Das wolle er auf keinen Fall, alle würden dann denken, es sei wegen des Ausweises gewesen. Auch hält er die Beziehung gegenüber seiner afghanischen Verwandtschaft geheim. Er versuchte sich schon verschiedentlich von der Freundin zu trennen oder zumindest auf Distanz zu gehen. Selina könne ihm niemals die Ehefrau werden, die er sich wünsche.

Vermutlich vermisste Herr $\mathrm{H}$. in dieser Beziehung die ihm gewohnte Art von Geschlechterordnung und Rollenteilungen. Nachfolgend ein Auszug aus meinen Einträgen, der seine diesbezüglichen Konflikte illustriert:

In der Schweiz stelle sich für ihn das Problem, wie er zum einen eine afghanische Frau finden könnte, und zum anderen, wie er wissen würde, dass sie gut sei und aus einer guten Familie stammen würde. Auch wäre es unmöglich, Selina mit nach Afghanistan zu nehmen. Als eine sich mit Kunst befassende Frau könnte sie dort nicht leben. Er habe das Gefühl, dass sie ihn, als Afghanen, einfach ausprobiert habe. Hier würden alle etwas machen, weil es Spass macht, er würde Dinge machen, weil er anderen helfen wolle.

Die Therapie mit Herrn H. hörte relativ abrupt mit der Bitte, dass er sich wieder an mich wenden könnte, wenn es ihm schlecht ginge, auf. Er müsse die Stunde absagen, da ibn ein Freund brauche, dessen Beziehung in die Brüche gegangen sei.

Vermutlich konnte die Psychotherapie Herrn H. eine gewisse Entlastung geben. Der psychotherapeutische Raum wurde zu einem Ort, wo er sein Heimweh aufleben lassen konnte. Er konnte thematisieren, was ihm alles fehlte, worin sich sein neues vom alten Leben unterscheidet. In Bezug auf seine Liebesbeziehung denke ich, dass es ihn unbewusst belastete, die Schweizer Freundin nicht heiraten zu wollen, und er dennoch deren Zuwendung genoss, was er aus «kulturellen» Gründen nicht gutheissen konnte. Es war, als ob er sie missbrauchte, sie «entjungferte», ohne sich dann um sie zu kümmern. Hinzu kommt, dass er sie nicht versorgen konnte, sondern umgekehrt von ihr versorgt wurde, und vor dem Hintergrund seiner Rollenbilder somit abhängig war wie eine Frau.

Herr H. fand schliesslich eine innere Haltung zu seiner Liebesbeziehung - eine voreheliche Zwischenlösung, wie es in der Schweiz üblich sei. Zudem bereitete er sich minutiös auf das zweite Gespräch mit den Migrationsbehörden vor in der Hoffnung, dass eine stringente Fluchtgeschichte, auch ohne Beweise, zu einem positiven Asylentscheid führen würde und ihm auf diese Weise das Heiraten erspart bliebe. Nicht zuletzt konnte er auch anerkennen, dass ihm seine Schweizer Freundin trotz ihres nicht-muslimischen Hintergrunds durch ihre emotionale Zugewandtheit eine Brücke zu einem neuen Leben in der Schweiz schlug.

\section{Verletzungen der islamischen Normativität}

Frau M. war bei Therapiebeginn 37 Jahre alt. Wir sprachen in den Sitzungen der gut zweieinhalbjährigen Psychotherapie französisch miteinander. Die Sunnitin stammte aus Algerien. Sie wechselte zu mir, als ihre erste Psychotherapeutin wegen eines Mutterschaftsurlaubs pausierte. Ich hatte keine grossen Vorinformationen zur Krankheitsgeschichte von Frau M. erhalten. Im Erstgespräch wurde klar, dass ihr Leiden als posttraumatische Belastungsstörung mit einer ausgeprägten Schmerzsymptomatik eingeordnet werden könnte. Auffallend waren Schilderungen, die auf eine dissoziative Symptomatik schliessen liessen: Frau M. stiess sich oft irgendwo an oder verbrannte sich und merkte dies erst hinterher. Weiter litt sie auch an einer Tic-Störung, ein Zucken eines Mundwinkels, dessen Ursachen - ob durch Psychopharmaka bedingt - nicht geklärt werden konnte. Rückblickend würde ich die therapeutische Arbeit als Hilfe bei einem 
Emanzipationsprozess einer Frau bezeichnen, die, im traditionellen algerischen Milieu aufgewachsen, schon früh auf Eigenständigkeit und Autonomie pochte, und die sich in der Schweiz nach einem äusserst schwierigen und konfliktreichen Start diesem Anspruch auf ein selbstbestimmtes Leben wieder nähern konnte.

Frau M. ist in einer mittelgrossen Stadt gross geworden. Sie hat einen Patchwork-Familien-Hintergrund, über den sie in der Therapie nur selten etwas erzählte. Unüblich zu den islamischen Gepflogenheiten lebte sie nach der Scheidung ihrer Eltern mehrheitlich bei ihrer Mutter, die einen religiösen zweiten Mann geehelicht hatte. Auch der Vater, den sie manchmal besuchte, war eine neue Ehe eingegangen. Frau M. hat mehrere Brüder und Halbbrüder. Ihre engste Beziehung hat sie zur zehn Jahre jüngeren Halbschwester. Ihre Schwester, ihre Mutter und sie seien sich sehr nah gestanden.

Bis zu ihrer Ausreise in die Schweiz führte sie ein Schneidereigeschäft. Sie war gelernte Schneiderin, dank einer Ausbildung, die sie ohne Wissen von Vater und Brüdern sich selbst finanziert hatte. Sie hatte Pläne, mit einem Kollegen, eine Ausbildungsstätte für Schneider*innen zu eröffnen. Als 32-Jährige lernte sie übers Internet einen Schweizer Mann kennen. Er machte ihr den Hof, kam sie besuchen, sie verliebte sich. Er war ihr erster Liebhaber. Alle Avancen von Männern hatte sie zuvor abgelehnt, da sie wusste, dass eine Heirat bedeutet hätte, ihre beruflichen Freiheiten zu verlieren. Sie hätte Touristenpaare, die so anders miteinander umgehen, gesehen. Der Mann verbrachte mehrmals die Ferien in Algerien. Er hielt um ihre Hand an, was ihre Mutter jedoch ablehnte, da er kein Muslim war. Frau M. reiste schliesslich mit Heiratsvisa und Rückkehrticket in die Schweiz - ohne familiären Segen.

Aus der Hoffnung auf ein freies Leben und eine respektvolle Ehe wurde für Frau M. jedoch ein Gräuel. Sie lernte in der Schweiz eine völlig andere Seite ihres Mannes kennen. Er habe sie geschlagen, gedemütigt und sexuell missbraucht. Wie eine Sklavin gehalten. Sie vermutete, dass er sich an ihr für seine Erfahrungen mit seiner ersten Ehefrau, einer Italienerin, die ihn betrogen und sich von ihm getrennt hatte, rächte.

Frau M. floh schliesslich nach knapp fünf Monaten, nicht wissend, dass sie schwanger war. Sie fand Schutz in einem Frauenhaus. Sie erhielt Unterstützung durch die Opferhilfe und zeigte ihren Ehemann an. Das Kind trieb sie, nach langem hin und her, ab. Sie suchte sich Arbeit in einem Lebensmittelgeschäft als Verkäuferin und als Putzfrau.

Sukzessive bildeten sich in den Monaten danach immer mehr, laut Ärzt*innen, psychosomatische Schmerzen heraus, zuerst im Bauch, dann im Rücken, über das Gesäss in die Beine laufend, wie Messerstiche. Sie konnte deswegen nicht still sitzen. Dazu kamen häufige Kopfschmerzen. Die für Frau M. jedoch schlimmste Folge war, dass ihre Familie aufgrund ihrer Eheschliessung - der Islam gestattet Musliminnen keine Ehe mit Nicht-Muslimen, vice versa jedoch schon ${ }^{7}$ - jeglichen Kontakt zu ihr abgebrochen hatte. Die Heirat sei für ibre Familie wie ein Erdbeben gewesen. Noch heute verbiete die Mutter ihrer Schwester, ibren Namen zu nennen. Frau M. lebte in der Angst, dass zwei ihrer Brüder einen Ehrenmord an ihr begehen würden, sie hätten ihr dies angedroht. Da sie beruflich als Gastarbeiter zeitweise in Frankreich zu tun hatten, fürchtete sie, dass ihr Wohnort bekannt werden könnte. Sie habe eine Sonderrolle zugestanden bekommen durch ihre Brüder. Sie hätten Vertrauen in sie gehabt. Sie habe dieses verspielt. Sie hätte nicht auf ihre Schweizer Liebe mit seinen Aussagen "es ist doch deine Sache, wen du liebst» hören sollen.

Eine weitere Folge der Heirat von Frau M. war, dass ihre Halbschwester zu einer Ehe gezwungen wurde. Sie musste ihr Studium abbrechen und konnte ihren Traum, Anwältin zu werden, nicht mehr verwirklichen. Frau M. hatte massive Schuldgefühle deswegen. Die jüngere Halbschwester war die einzige, mit der sie manchmal heimlich telefonierte.

Rund zweieinhalb Jahre nach ihrer Ankunft in der Schweiz begann ihre Psychotherapie bei mir. Wegen ihrer Schmerzen setze sie sich für die Therapiesitzungen auf meine Liege, mit vielen Kissen gepolstert, und nicht auf einen Sessel. Inhaltlich ging es im ersten Jahr der Psychotherapie um viele Themen: Wut angesichts der Freisprechung ihres Mannes, in dubio pro reo - trotz Krankenhausberichten, die Verletzungen an der Vagina und im unteren Rückenbereich beschrieben. Sie stellte sich den Erinnerungen an die erzwungenen Sexualakte, das Gefühl, dass ein Tier ihren Körper verletzt habe. Es ging um die Aufarbeitung der Schuldgefühle ihrer Schwester und dem abgetriebenen Kind gegenüber. Sie bereute ihren Entscheid, das Kind, das in der Schweiz zu einem Familienersatz hätte werden können, abgetrieben zu haben. Die Therapiegespräche drehten sich um den Verlust ihrer Familie, ihren Selbsthass und die Angst, dass ihr durch ihre Familie etwas geschehen könnte. Frau M. sah sich im Internet verschiedene Reportagen zu Ehrenmorden an und weinte angesichts der Parallelen, die sie dort zu ihrer eigenen Geschichte entdeckte. Belastend kam hinzu, dass sie Angst hatte, aus der Schweiz ausgewiesen zu werden. Sie war nicht lange verheiratet gewesen und musste bangen, dass ihre fünfjährige Aufenthaltsbewilligung nicht erneuert werden würde. Eine Rückkehr nach Algerien wäre ihrem Empfinden nach einem Todesurteil gleichgekommen. Mit eisernem Willen zwang sie sich trotz ihrer Schmerzen an ihrer Arbeitsstelle auszuharren, da sie wusste, dass ihr ohne Arbeit ein Bleiberecht leichter aberkannt werden könnte.

Im Verlauf der Psychotherapie nahmen ihre heftigen Albträume ab. Ihre Schmerzen begannen sich etwas zu mildern. Es klärten sich Bezüge der Schmerzen zu lebensgeschichtlichen Ereignissen. Ob die Tic-Störung ihres Mundwinkels damit zu tun haben könnte, dass ihr Ehemann, wie Frau M. geschildert hatte, sie als Muslimin gezwungen habe, vor anderen Wein zu trinken, und ein-

7 Hintergrund dieses Verbots ist, dass Kinder in der Regel den Glauben des Vaters übernehmen. 
mal habe er ihr Reis ins Gesicht geschmiert, erwäge ich erst jetzt beim Schreiben dieser Fallvignette als mögliche Erklärung. Auch ihre massiven Ängste, wenn sie nachts unterwegs oder allein in ihrer Wohnung war, verringerten sich. Es gab Tage, an denen sie die Einnahme der Schmerzmedikamente reduzieren konnte.

Frau M. begann die Seite in sich, die lebenslustig für ihr Recht auf Arbeit, Beziehung und Sexualität eingetreten war, wieder mehr zu schätzen. Sie ging soweit, dass sie sich auf einen Liebesabend mit dem Besitzer des Lebensmittelladens, einem älteren Muslim, ein verheirateter Vater eines Sohns, der schon lange versuchte mit ihr anzubandeln, einliess. Womit sie nicht gerechnet hatte: Sie wurde erneut schwanger. Im Nachhinein erklärte sie mir dazu, dass meine Frage, ob sie nicht auch interessiert sei herauszufinden, ob es eine schöne Art von Sexualität geben könnte, den Wunsch bei ihr geweckt hatte, dies auszuprobieren. Vielleicht war es auch das unbewusste Motiv, eine Wiederannäherung an ihren Vater zu finden, der sich nicht gross um sie gekümmert hatte und phasenweise in Frankreich als Gastarbeiter lebte. Frau M. entschied, das Kind zu behalten. Die Schwangerschaft versetzte sie in eine euphorische Stimmung angesichts der Vorstellung, dass sie nicht mehr allein sein würde. Im Hinterkopf sammelte sich aber auch die Erkenntnis, dass dieser Umstand einen erneuten Brückenschlag zu ihrer Familie unmöglich machte, da sie wohl als Ehebrecherin betrachtet werden würde.

Die kurze Blütephase hielt nach der Geburt nicht lange an. Psychisch labil als alleinerziehende Mutter für ein Kind da zu sein, war anstrengend, trotz der Unterstützung einer guten Freundin. Dazu kamen die inneren Konflikte und Selbstzweifel: Es sei haram, gegen ihre Religion, was sie gemacht habe. Von der muslimischen Frau des Vaters ihres Kindes wurde sie als Schlampe beschimpft. Frau M. begann es wieder schlechter zu gehen. Überforderungsgefühle machten sich breit. Sie entwickelte ambivalente Gefühle der Tochter gegenüber. Zu den inneren Konflikten kamen äussere hinzu: Der Chef stritt ab, der Vater zu sein, die algerischen Behörden taten sich schwer mit einem unehelichen Kind. Sie setzt sich in dieser Zeit in den Therapiesitzungen oft vor mir auf den Boden, vordergründig angesichts ihrer Schmerzen. Ich hatte das Gefühl, sie bitte um Absolution, dass ich ihr, stellvertretend für alle anderen, verzeihe.

Allmählich begann sich Frau M. wieder zu fangen. Es gelang ihr, sich Hilfe zu organisieren. Sie setzte juristisch durch, dass ein Vaterschaftstest gemacht wurde, welcher die Vaterschaft erhärtete. Sie erhielt eine Verlängerung ihrer Aufenthaltsbewilligung. Sie kämpfte für die Zurücknahme des gemeinsamen Sorgerechts, da dieses für sie viele Dinge komplizierte. Die Psychotherapie wurde in diesen Monaten auch ein Ersatzfamilienraum. Frau M. brachte die Tochter alle paar Wochen mit in die Stunden. Ich bestaunte das Drehen, das Krabbeln, die ersten Schritte, die ersten Worte, und ich freue mich mit ihr über den gefundenen Kitaplatz in der Nähe ihrer Wohnung.

Frau M. beendete die Psychotherapie nachdem sie erfuhr, dass die algerischen Behörden ihre Tochter end- lich akzeptierten. Sie werde nun einen Pass für ihr Kind erhalten. Eine grosse Last sei von ibren Schultern gefallen. Es war die letzte gemeinsame Sitzung. Danach erhielt ich nur noch Anrufe auf Band, dass sie sich wieder melden würde. In dieser Sitzung hatte eine entspannte Frau vor mir gesessen. Sie sprach von Entwicklung und Fortschritt: Sie habe sich verändert seit ibrer Ankunft in der Schweiz. Am Anfang in der Schweiz sei sie die Fremde gewesen. Nun kenne sie sich aus. An der Kasse habe die Verkäuferin letzthin mit ihr nur deutsch geredet. Sie trete viel selbstbewusster auf. Sie wünsche sich, dass auch ibre Tochter so selbstbewusst werde wie sie. Es sei schön zu sehen, dass Kinder in diesem Alter noch keine Angst vor der Welt hätten.

\section{Konversion zum Islam als Lösungsversuch}

Bei der Psychotherapie mit Frau T. handelt es sich um eine laufende Behandlung. Die nachfolgende Fallbeschreibung ist daher als vorläufig zu betrachten, eine der möglichen Narrationen, die zu dieser Patientin gemacht werden könnten. ${ }^{8}$ Mich erinnert die Lebensgeschichte von Frau T. an Ausführungen des Ethnopsychoanalytikers Tobie Nathan (2017), die er in seinem Buch über die islamische Radikalisierung junger Menschen in Frankreich macht. Ich möchte ihren Fall vor dem Hintergrund seiner Überlegungen vorstellen. Ich gehe dabei vor allem auf die Vergangenheit von Frau T. ein.

Über ihre Lebensgeschichte weiss ich aufgrund der Schwere ihrer Erkrankung, einer paranoiden Schizophrenie, nur Bruchstücke. Sie stammt aus Eritrea und ist die zweitjüngste von mehreren Geschwistern sowie Tochter eines orthodoxen christlichen Pfarrers. Er sei ein weiser Mann gewesen. Einzig, dass er gegen Muslime war, habe sie gestört. Frau T. war bei Therapiebeginn 26 Jahre alt. Sie schätzte den psychotherapeutischen Raum. Es tue ihr gut, wenn sie erzählen könne, was sie beschäftige. Es ist eine Psychotherapie, die dolmetschergestützt verläuft. ${ }^{9}$

Frau T. wurde von den Mitarbeitenden ihrer Asylunterkunft für eine Gruppenpsychotherapie in unserem Zentrum angemeldet: Sie wirke depressiv und angeschlagen. Zu mehr als Indikationsgesprächen kam es jedoch nicht, da Frau T. infolge einer psychotischen Dekompensation mehrere Monate in einer psychiatrischen Klinik stationär behandelt werden musste. Noch aus der Klinik meldete sie sich bei mir und bat um Hilfe. Die Tatsache, dass ich basale Kenntnisse des Arabischen habe, einer Sprache, die sie gelernt hatte, mag dabei eine Rolle gespielt haben.

Nach Klinikaustritt begann sie bei mir eine Einzeltherapie. Aufgrund ihres Wahns waren die Psychotherapiesitzungen mit ihr im wahrsten Sinne des Wortes «ver-rückt», es ging um den Brückenschlag zu ihrer Privatwirklichkeit und der Begegnung dreier Menschen im Hier und Jetzt

8 Freud (1912e) zufolge kann erst nach Behandlungsabschluss effektiv gesagt werden, worum es sich eigentlich gehandelt habe.

9 Zuerst im Rahmen eines Pilotprojekt des Bundes, später über Gesuche bei Gesundheitseinrichtungen. 
(vgl. Rom, 2007). ${ }^{10}$ Anamnestische Erkundigungen konnten nur beiläufig gemacht werden. Der Dolmetscher war nach jeder Sitzung müde und geschafft, da Frau T. oft auf Fragen vorbeiantwortete und mit ihrer paranoiden Welt- und Beziehungswahrnehmung unsere Kommunikation einhüllte. Über ihre Kindheit liess sie uns wissen: Sie sei nicht gerne zur Schule gegangen. Sie habe es stets vorgezogen, Schafe zu hüten. Ihr Vater habe sich, nachdem sie aus der Hauptstadt aufs Land gezogen waren, eine Schafherde zugelegt. Sie sei für diese verantwortlich gewesen: mindestens 50 Tiere. Sie sei eine gute Hirtin gewesen. Zwei muslimische Hirtenmädchen seien ibre besten Freundinnen gewesen. Schon als Kind habe sie viele Träume gehabt, zum Beispiel, dass ein Kamel käme und sie weghole; oder auch, dass andere Kinder mit Steinen nach ihr werfen würden. Es seien spezielle Träume gewesen, als ob diese wahr seien. Auch jetzt, denke sie, dass das, was sie träume, wahr werde.

Mit zwölf Jahren wurde sie zwangsverheiratet mit einem älteren Mann, gegen ihren Willen. Die Mutter von Frau T. war gegen die Ehe gewesen, hatte dann aber den Entscheid ihres Mannes und der älteren Söhne akzeptiert. Mit 14 Jahren bekam sie ihr erstes Kind, kurz später ein zweites, das jedoch kurz nach der Geburt starb. Frau T. verliess schliesslich ihren Ehemann. Sie sei dort nicht gut behandelt worden, ihr Mann habe sie geschlagen. Sie liess ihre Tochter zurück und ging zurück in ihre Herkunftsfamilie. Wenig später verstarb ihre Mutter. Sie verlor damit eine wichtige Unterstützungsperson. Aus den fragmentierten Erzählstücken der verschiedenen Therapiesitzungen, die ohne lineare Zeitabfolge geschildert werden - oft belässt es Frau T. zudem mit einem «es sind schlimme Dinge passiert» - lassen sich folgende Geschehnisse rekonstruieren: Frau T. migrierte in das Nachbarland Sudan. Anscheinend besass sie etwas Gold und Schmuck. Sie lernte einen muslimischen Mann kennen, den sie heiratete und mit dem sie zwei weitere Kinder bekam. Dank ihrer Finanzen hätten sich ihr Mann und sie ein Haus leisten können. Aufgrund von Eheproblemen und möglicherweise auch einer schweren Erkrankung des Vaters wollte Frau T. nach Eritrea zurückkehren. Sie drängte ihren Mann, das gemeinsame Haus zu verkaufen oder ihr zumindest einen Teil des Geldes zurückzugeben, den sie für den Hauskauf beigesteuert hatte. Sie konnte dies jedoch nicht beweisen, da ihr Mann als Besitzer des Hauses eingetragen war. Sie versuchte sich bei der eritreischen Botschaft Hilfe zu organisieren, was jedoch misslang. I $h r$ Mann habe ihr schliesslich den Pass weggenommen. Diese Ereignisse müssen rund um die Geburt des zweiten Kindes aus dieser Ehe passiert sein. Vermutlich litt Frau T. bereits in jener Zeit an ihrer paranoiden Erkrankung, denn sie wurde auch im Sudan psychiatrisch und medikamentös behandelt. Frau T. zufolge erschien ihr kurz danach ein weisses Pferd, das sie umarmte und ihr mitteilte, dass sie Muslimin werden solle. Sie konvertierte. I hr Mann habe

10 Rom entwickelte ausgehend von Benedetti ein Konzept für Psychotherapien mit psychotischen Menschen. Grundidee ist die Entwicklung eines dualen Raums. ihr das nicht geglaubt, dass sie eine Erscheinung gehabt habe, auch andere Leute nicht. Aber die Leute würden dann doch nachdenklich werden, wenn sie von dieser Erfahrung im Detail erzäble.

Wieso Frau T. als 24-Jährige in die Schweiz kam, liegt im Dunkeln. Eine Vermutung ist, dass sie aufgrund ihrer sich schon im Sudan zeigenden Erkrankung für eine bessere Behandlung nach Europa «abgeschoben» wurde, da sie der Familie zur Last fiel. Sie selbst berichtete, dass man in der Moschee ihres Wohnorts Geld gesammelt habe, um ihr die Reise zu ermöglichen. Sie habe für die Schlepper 3000 US-Dollar gezahlt. Eine reiche Fran habe den Rest beigesteuert, eine gute Frau. Sie hätte nie damit gerechnet, dass sie in die Schweiz käme.

Die grösste Sorge von Frau T. in den zwölf Monaten ihrer Psychotherapie bei mir war, dass man sie wegen ihres Konvertierens verfolgen würde. Man sei ibr missgünstig gesonnen. Man habe sie schon im Sudan zu vergiften versucht. Sie habe monatelang kein Wasser lösen können. Sie habe gezittert und ihr Herz habe geschlagen. Hier in der Schweiz würden sie grosse schwarze Vögel warnen, wenn ihr Gefahr drohe. Sie würden mit ihr sprechen. Manchmal seien sie sehr aufgeregt. Über meine Praxis hätten die Vögel gesagt, dass dies ein guter Ort sei. Es gebe auch kleinere Vögel, mit schwarz-weissen Federn. Sie kämen an ibr Fenster. Die Leute seien manchmal neidisch, dass sie mit Vögeln sprechen könne. Fotografieren könne sie sie nicht, das gehe nicht, das habe sie schon versucht. Meine Besorgnis wahrnehmend versicherte sie mir, dass diese Vögel für sie gut seien. Sie seien für sie wie Schutzengel. Auch im Koran seien solche Vögel erwähnt. Sie sei nicht krank. ${ }^{11}$

Die Psychotherapiesitzungen mit Frau T. waren schwindelerregend, da sich reale Probleme bzw. wahnhaft von ihr interpretierte Ereignisse mit Verfolgungs- und Vergiftungsvorstellungen vermischten. Real war zum Beispiel, dass es in ihrer Asylunterkunft Konflikte mit Mitbewohner*innen gegeben hatte, wenn sie auf Youtube laute Rezitationen von Koransuren hörte. Wahnhaft interpretiert war es, wenn sie dachte, dass man ihr Teile des Monatsgelds vorenthalte und der Abzug jedoch durch andere Auslagen bedingt war. Wahnhaft war ihre Vorstellung, aufgrund ihrer Konversion in die Ungnade des eritreischen Präsidenten gefallen zu sein. Frau T. witterte phasenweise Beziehungen und Verbindungen zwischen allen. Aus der Ferne könnten Menschen bewirken, dass sie Schaden nehme, mit Zauberei. Sie sei zum Glück geschützt durch ihre Gebete. Ihr Glaube lasse sie wissen, dass dies auf diejenigen, die etwas Böses tun, zurückfallen werde. Sie war besorgt und gleichzeitig getröstet, da sie alle schwie-

11 In islamischen Gesellschaften gibt es eine höhere Prävalenz von visuellen Halluzinationen mit kulturspezifischen Inhalten. Al-Issa (2000, S. 330) diskutiert bspw., ob die WHO-Diagnosekriterien einer Schizophrenie für Menschen aus kollektivistischen Gesellschaften angebracht seien. Die Tatsache, Gestalten wahrzunehmen, sei noch kein Krankheitssymptom an und für sich, insbesondere dann, wenn deren Existenz normativ akzeptiert sei und die Fähigkeit, mit der spirituellen Welt in Kontakt zu treten, mit Ehrfurcht betrachtet wird. 
rigen Vorkommnisse als Gottesprüfungen einordnete, die sie, da Gott über ihr wache, unbeschadet überstehen würde. Den Klinikaufenthalt hatte sie wegen der erfolgten Zwangsmedikation in schlechter Erinnerung und sah auch dort ein gegen sie gerichtetes Komplott. Frau T. hatte die Medikation nach Klinikaustritt sofort gestoppt. Auch wie sie mich einschätzen sollte, beschäftigte sie: Man sage ihr, dass ich ibr nicht gut gesonnen sei, sie solle zu einer besseren Ärztin wechseln. Aber sie wisse, ich sei jemand Gutes, so formulierte sie ihre Ambivalenzen mir gegenüber.

Aktuell wird Frau T. wieder stationär behandelt. Ein Jahr lang war sie relativ stabil gewesen. Ihre paranoiden Ängste spitzten sich jedoch im Zusammenhang mit sozialem Druck von eritreischen Bekannten und Verwandten zu. Es wurde von ihr erwartet, sich vom Islam zu distanzieren.

Wie ist nun die Konversion und Erkrankung von Frau T. zu verstehen? Nathan (2017) arbeitet in seinem Buch Les Âmes Errantes heraus, weshalb sich junge in Frankreich aufgewachsene Secondos vermehrt der muslimischen Religion zuwenden, sogar wenn diese aus nicht-muslimischen Familien stammen. Folgende These leitet seine Überlegungen: Dadurch würden sämtliche Probleme dieser in aller Regel jungen, kulturell heimatlos gewordenen «Seelen», eine Zweitgeneration von Migrant*innen, beseitigt werden. Durch die Wiederentdeckung des Islam oder die Konversion zum Islam würden psychologische Konfusionen rund um die eigene Identität oder Zugehörigkeit gelöst werden. Es seien vielfach Ersatzhandlungen für individuelle Schwierigkeiten rund um den Verlust einer gefühlten und gelebten Zugehörigkeit und Herkunft, manchmal gar mit psychopathologischem Gehalt, die seit Kindheit bestanden hätten, und die nun mit einem Mal geklärt und gelöst werden. ${ }^{12}$ Aus Unwissen und Orientierungslosigkeit werde Gewissheit und eine Einbindung in die Welt. Aus diesem Umstand bezöge die «brutale Metamorphose» eine Kraft. Weder Vernunft, Einspruch noch Argumente könne diese nach aussen oft durchschnittlich wirkenden jungen Erwachsenen wieder davon abbringen:

«Eine Zeitverschwendung, weil ihr Konvertieren kein Problem, kein Symptom, sondern eine Lösung ist! [...] Die Kraft der Offenbarung, die mir dieser Art von Bekehrung einhergeht, ist nicht zu übersehen, als ob ein Schleier vor den Augen des Menschen zerrissen würde [..., ein Gefühl, dass dann alles erklärt werden kann, der eigene $\mathrm{Zu}$ stand und der der Welt» (ebd., S. 88; Übers. d.A.).

Der Kniff bestände darin, dass die Rätsel der Vergangenheit rund um die eigene Identität und Zugehörigkeit durch diese einfache Kehrtwende zu einem gestaltbaren Rätsel der Zukunft werde: zur Entdeckung einer Wahrheit über sich und die Welt (ebd., S. 86ff.).

12 Nathan benutzt für die Herkunft und Zugehörigkeit eines Menschen die Metapher der «Quelle» im Unterschied zu statischen «Wurzeln»: «Bei den Menschen erneuert sich die Herkunft unablässig; denn für sie ist die Herkunft nicht ein Instinkt, sondern zugleich Wissen und Wille» (ebd., 2017, S. 59; Übers. d. A.).
Ich möchte die Konversion meiner Patientin nicht überstrapazieren. Doch mit Bezugnahme auf die Konzeptualisierungen von Mentzos (2013) und Benedetti (1980), die Wahnerkrankungen als in der Biografie eines Menschen zu verortende funktionale Bewältigungsversuche von massiven Ängsten und diffusen innerpsychischen Spannungen in Dilemmasituationen betrachten, könnte die durch eine Erscheinung motivierte Konversion von Frau T. auch als Ersatzhandlung im Sinne eines Identitätsbildungsversuchs einer «herumirrenden Seele» betrachtet werden. So führte Frau T. auch in einer Sitzung aus: Ihr sei eine Lichtgestalt erschienen, die ibr mitgeteilt habe, dass sich ibr Vater mit ibr wieder versöhnt habe und akzeptiere, dass sie Muslimin geworden sei.

\section{Schlussbemerkung}

Die drei Fallbeispiele zeigen die Diversität von muslimischen Patient*innen in der psychoanalytischen Praxis auf. Sie verdeutlichen, dass Fragen der Religiosität im Allgemeinen eingebettet sind in die der Kultur, der die Patient*innen entstammen. Die islamische Normativität ist immer Teil der nationalen oder ethnischen Kultur der Patient*innen - in sie eingewoben und eingeflochten. Anders ausgedrückt: Die national-ethnische Kultur überlagert die religiöse. Gerade das erste Fallbeispiel zeigt die situationsbezogenen, wechselnden Identifikationen des Patienten, der sich je nach Kontext mir als Psychotherapeutin mal mehr als Afghane, mal mehr als Muslim, mal mehr als Paschtune präsentiert.

Gleichzeitig sind die drei Fallbeispiele auch Migrationsgeschichten, die sich in einer globalisierten Welt ereignen. Das psychoanalytische Setting bietet den Rahmen, den Verlust der verlorenen Umwelt-Mutter zu betrauern und sich mit Triebregungen, die im Unterschied zur Herkunftskultur erlaubter oder tabuisierter sind, zu beschäftigen (Saller, 2016). Im zweiten Fallbeispiel erhellte sich zudem, dass alte Konfliktlagen im neuen soziokulturellen Kontext aktualisiert werden und erneut gelöst werden müssen: Die muslimische Patientin hatte bereits in ihrer Heimat einen unkonventionellen weiblichen Lebensweg eingeschlagen und sich an den Rändern der islamischen Normativität bewegt. Das Beispiel könnte als Illustration der von Parin (1978) formulierten Theorie des Widerspruchs im Subjekt betrachtet werden. Es veranschaulicht den Einfluss der begehrenden unbewussten psychischen Landkarte, die lebensgeschichtliche Entscheide im Lebensverlauf mitformt. Ebenso macht es begreiflich, wie eine Abkehr von Rollen-Identifikationen, die sich wie ein Fremdkörper im Ich eingenistet haben, gelingen kann, zum Preis, dass sich das Ich neu orientieren und andere Formen von Gratifikationen finden muss. Im dritten Fallbeispiel beschrieb ich die radikale Hinwendung zum Islam als identitätssuchende Zugehörigkeitskonstruktion, die als Ersatzhandlung tiefgreifendere innere Konfusionen der Patientin zu besänftigen versucht: gemäss Nathan (2017) ein Lösungsversuch für identitätsbezogene Schwierigkeiten von kulturell heimatlos gewordenen jungen Menschen. In 
keiner der drei Psychotherapien wurde die unterschiedliche Religionszugehörigkeit zwischen Patient" in und mir als Psychotherapeutin intensiver angesprochen. Alle drei Patient"innen nahmen mir gegenüber die Position ein, dass ihre islamisch andere Form des Glaubens grundsätzlich an einen uns gemeinsamen Gott gebunden sei. Mein kulturelles Nicht-Wissen bot ihnen die Gelegenheit der Selbstreflexion und damit auch der Bearbeitung und Veränderung ihrer persönlichen Vorstellungen und innerpsychischen Ambivalenzen rund um ihre religiösen wie kulturellen Handlungen, Wünsche und Normen. Sie profitierten vom psychoanalytischen Prozess, indem sie aus den sie umtreibenden Erfahrungen bewusste und damit mit sich verhandelbare Probleme bildeten.

Der Boden für psychoanalytische Psychotherapien mit muslimischen Migrant*innen ist im Westen vielleicht doch fruchtbarer als gedacht. Da sich ihre lebensweltlichen Umstände durch die Migration verändert haben, werden sie auf jeden Fall mit vielfältigen individuellen und singulären Erfahrungen konfrontiert. Anders als in islamischen Gesellschaften, wo die klassische Psychoanalyse oft nicht richtig greifen kann, bietet dies möglicherweise den Ansatzpunkt dafür, dass das psychoanalytische Heilungsritual als gewinnbringend erlebt wird. In der Auseinandersetzung mit sich und dem suchenden und begehrenden widersprüchlichen Innenleben finden die Patient"innen neue Balancen. Die zu Beginn einer Therapie mit Patient*innen aus einer anderen Kultur bestehende gegenseitige Deutungsohnmacht - man nimmt Unterschiede wahr und kommt mit den gewohnten automatisierten Erklärungsmustern nicht weiter - kann Angst machen; die wahrgenommenen Differenzen und «weissen Wände», die nicht beschrieben und bebildert werden können, schüchtern ein. Der Reiz von Psychotherapien mit (muslimischen) Migrant*innen besteht jedoch darin, dass nach der anfänglichen auszuhaltenden transkulturellen «Sprachlosigkeit» mit der Zeit in einem gemeinsamen kreativen Akt im Unbekannten wieder Bekanntes entdeckt werden kann (Saller, 2003, S. 419ff.).

\section{Literatur}

Akhtar, S. (2008). Introduction. In S. Akhtar, The crescent and the couch. Cross-currents between islam and psychoanalysis (S. xiiixix). Lanham: Jason Aronson.

Al-Abdul-Jabbar, J. \& Al-Issa, I. (2000). Psychotherapy in islamic society. In I. Al-Issa (Hrsg.), Al-Jun n: Mental illness in the islamic world (S. 277-293). Madison: International Universities Press.

Al-Issa, I. (2000). Does the muslim religion make a difference in psychopathology? In I. Al-Issa (Hrsg.), Al-Jun n: Mental illness in the islamic world (S. 315-353). Madison: International Universities Press.

Allenbach, B. (2019, i. D.). Migration und Agency: Wie Jugendliche auf Prozesse des Andersmachens reagieren. In J. Hangartner, M. Jäger, M. Kuhn, A. Sieber Egger \& G. Unterweger (Hrsg.), Zwischen institutioneller Regulierung und Selbstbestimmung: Ethnographische Beiträge zu Kindheit(en) in der Schweiz. Wiesbaden: Springer VS.

Allenbach, B. \& Herzig, P. (2010). Der Islam aus Sicht von Kindern und Jugendlichen. In B. Allenbach \& M. Sökefeld (Hrsg.), Muslime in der Schweiz (S. 296-330). Zürich: Seismo.

Allenbach, B. \& Sökefeld, M. (2010). Einleitung. In B. Allenbach \& M. Sökefeld (Hrsg.), Muslime in der Schweiz (S. 9-40). Zürich: Seismo.
Amnesty International, Schweizer Sektion (27.11.2017). Syrische Flüchtlinge in der Schweiz. Zahlen, Fakten und Hintergründe. https:// www. amnesty.ch/de/themen/asyl-und-migration/asylpolitik-schweiz /dok/2017/syrische-fluechtlinge-in-der-schweiz (06.01.2019).

Ansary, T. (2010). Die Unbekannte Mitte der Welt. Globalgeschichte aus islamischer Sicht. Frankfurt a. M.: Campus.

Azhar, M.Z. \& Varma, S.L. (2000). Mental illness and its treatment in Malaysia. In I. Al-Issa (Hrsg.), Al-Jun n: Mental illness in the islamic world (S. 163-186). Madison: International Universities Press.

Behloul, S. M. (2011). Unbeabsichtigte Folgen der Islam-Debatte. In Bulletin Schweizerische Gesellschaft Mittlerer Osten und Islamische Kulturen, SGMOIK-Bulletin, Muslime in der Schweiz, 32, 13-18.

Behloul, S. M. (2018). Islam. Muslimische Migranten in der Schweiz. Universität Luzern, Zentrum Religionsforschung. https://www. unilu.ch/ fakultaeten/ksf/institute/zentrum-religionsforschung/religionen-schweiz /religionen/islam (06.01.2019).

Benedetti, G. (1980). Klinische Psychotherapie. Einführung in die Psychotherapie der Psychosen. Bern: Verlag Hans Huber.

Birrer, R. (20.12.2017). Anzahl Muslime wird überschätzt. Tagesanzeiger, 20. Dezember 2017 (06.01.2019). https://www. tagesanzeiger.ch/ schweiz/standard/anteil-der-muslime-wird-ueberschaetzt/story/19819019 (06.01.2019).

Bleisch, P. (2016). Gelebte und erzählte Scharia in der Schweiz. Empirische Studien zur Aneignung religiöser Normen durch zum Islam konvertierte Frauen. Zürich: Schulthess.

Bundesamt für Statistik. (2014). Die Religion, eine Familiengeschichte. Analyse von Daten aus der Erhebung zur Sprache, Religion und Kultur 2014. https://www. bfs.admin.ch/bfs/de/home/statistiken/ bevoelkerung/sprachen-religionen/religionen. assetdetail.6087709.html (06.01.2019).

D’Amato, G. (2015). How Foreigners Became Muslims: Switzerland's Path to Accommodating Islam as a New Religion. In M. Burchardt \& I. Michalowski (Hrsg.), After Integration (S. 285301). Wiesbaden: Springer.

Dütschler, M. (2018). Lieber zu wenig tun als das Falsche. Der Bund, 11. Dezember 2018, 17.

El Khayat, G. (1994). Une psychiatrie moderne pour le Maghreb. Paris: L'Harmattan.

El Shakry, O. (2014). The Arabic Freud. The unconscious and the modern subject. Modern Intellectual History, 11(1), 89-118. https://www. academia.edu/6247448/The_Arabic_Freud_The_ Unconscious_and_the_Modern_Subject (06.01.2019).

Freud, S. (1912e). Ratschläge für den Arzt bei der psychoanalytischen Behandlung. GW VIII, 376-387. http://www. psyalpha.net/ themen/behandlungstechnik/freud-technische-schriften/sigmundfreud-1912e-ratschlaege-arzt-psychoanalytischen-behandlung (06.01.2019).

Geertz, C. (1983). Dichte Beschreibung. Beiträge zum Verstehen kultureller Systeme. Frankfurt a. M.: Suhrkamp.

Giani, M. (2016). Accommodating to Swiss religious pluralism: Interrogating Muslim integration and Swiss citizenship. In E. Aslan (Hrsg.), Islam, religions, and pluralism in Europe (S. 127-142). Wiesbaden: Springer VS.

Graef-Calliess, I. T. \& Schouler-Ocak, M. (Hrsg.). (2017). Migration und Transkulturalität. Stuttgart: Schattauer.

Hafner Al Jabaji, A. (2011). Das Schweigen der Ethnologie. In Bulletin Schweizerische Gesellschaft Mittlerer Osten und Islamische Kulturen, SGMOIK-Bulletin, Muslime in der Schweiz, 32, 24-26.

Hamad, N. (2004). Le sujet en islam. La célibataire (La Psychanalyse et le monde arabe), 8, 147-153.

Hamid, H. (2008). Basic history and tenets of islam. A brief introduction. In S. Akhtar (Hrsg.), The crescent and the couch. Cross-currents between islam and psychoanalysis (S. 3-19). Lanham: Jason Aronson.

Hammad, M. (2004). Le divan et l'oxydant. La célibataire (La Psychanalyse et le monde arabe), 8, 97-103.

Harari, Y.N. (2013). Eine kurze Geschichte der Menschbeit. München: Pantheon. 
Hartmann, K. (2007). Die Psychoanalyse im Libanon. Zur Entwicklung einer westlichen Profession in einer arabischen Gesellschaft. Berlin: Verlag Hans Schiller.

Hartmann, K. (2008). Psychoanalyse im Libanon. Zum Transfer der Psychoanalyse in ein arabisches Land, Werkblatt. Psychoanalyse \& Gesellschaft, 60, 107-127.

Hartmann, K. (2015). Psychoanalyse auf Arabisch. Journal für Psychoanalyse, 56, 66-82.

Herter, M., auf der Maur, N. \& von Wyl, H. (2015). Zahlen und Fakten zu Muslimen in der Schweiz. SRF. https:/www.srf.ch/news/ schweiz/jetzt-reden-wir/zahlen-und-fakten-zu-muslimen-in-derschweiz (06.01.2019).

Houbballah, A. (2004). La psychanalyse et le monde arabe. La célibataire (La Psychanalyse et le monde arabe), 8, 19-28.

Kader, H. A. (2004). La Psychanalyse en Égypte entre un passé ambitieux et un futur uncertain. La célibataire (La Psychanalyse et le monde arabe), 8, 61-73.

Khan, R. Y. (2008), Oedipus in Egypt: A Twentieth-Century Rendition of "Majnun Lalya». In S. Akhtar (Hrsg.), The crescent and the couch. Cross-currents between islam and psychoanalysis (S. 283294). Lanham: Jason Aronson.

Khalil, O. (2004). Pour une archéologie de la langue arabe. La célibataire (La Psychanalyse et le monde arabe), 8, 87-96.

Kakar, S. (1999). Psychoanalyse in nicht westlichen Kulturen. In F. Pedrina, V. Saller, R. Weiss \& M. Würgler (Hrsg.), Kultur. Migration. Psychoanalyse (S. 13-28). Tübingen: Edition diskord.

Magdi, A.H.S. (2004). La notion d'individu dans la culture arabe. La célibataire (La Psychanalyse et le monde arabe), 8, 161-169.

Mentzos, S. (2013). Lehrbuch der Psychodynamik. Die Funktion der Dysfunktionalität psychischer Störungen. Göttingen: Vandenhoeck \& Ruprecht.

Meier, M. (2018). Schlüsselfiguren der Integration. Tagesanzeiger, 2. April 2018. https://www. tagesanzeiger.ch/schweiz/standard/ schluesselfiguren-der-integration/story/26855272 (06.01.2019).

Melman, C. (2004). Remarques sur les conditions de possibilité de la cure. La célibataire (La Psychanalyse et le monde arabe), 8, 171-176.

Osseiran, M. (2010). De quelques difficultés de la pratique psychanalytique au Liban. Topique - Revue Freudienne, 110, 97-103.

Nathan, T. (1994). L'influence qui guérit. Paris: Éditions Odile Jacob.

Nathan, T. (2017). Les âmes errantes. Paris: L'iconoclaste.

Parin, P. (mit Parin-Matthèy, G.) (1978). Der Widerspruch im Subjekt. Die Anpassungsmechanismen des Ichs und die Psychoanalyse gesellschaftlicher Prozesse. In S. Drews et al. (Hrsg.), Provokation und Toleranz. Festschrift für Alexander Mitscherlich zum siebzigsten Geburtstag (S. 410-435). Frankfurt a. M.: Suhrkamp (http:// paul-parin.info/wp-content/uploads/texte/deutsch/1978d.pdf).

Rom, J. (2007). Identitätsgrenzen des Ich. Einblicke in innere Welten Schizophrenie- und borderlinekranker Menschen. Göttingen: Vandenhoek \& Ruprecht.

Safouan, M. (2004). Pratique analytique dans le monde arabe: incidences et difficultés. La célibataire (La Psychanalyse et le monde arabe), $8,11-18$.

Saller, V. (2003). Wanderungen zwischen Ethnologie und Psychoanalyse. Psychoanalytische Gespräche mit Migrantinnen aus der Türkei. Tübingen: Edition diskord.

Saller, V. (2016). Andere Kultur. Migration als Trauma. Unveröffentlichtes Referat in Bern.

Schweizerisches Zentrum für Islam und Gesellschaft (SZIG) (2018). Muslime im öffentlichen und medialen Raum, SZIG-Paper, 5. https://www3.unifr.ch/szig/de/assets/public/uploads/Recherche/A5 SZIG_Themenheft_5_D_WEB.pdf (06.01.2019).

Sheena (2018). Hearing Muslim Women's Voices at the Dergah. Jezebel, 7. August 2018. https://jezebel.com/hearing-muslim-womensvoices-at-the-dergah-1827753789 (06.01.2019).

Slama, R. B. (2010). La psychoanalyse en Égipte. Un problème de nonadvenue. Topique - Revue Freudienne, 110, 83-96.

Vincent, T. (2016). Dieu sans religion. Foi et démocratie. Fontaine: Presses universitaires de Grenoble.
Zeldin, K. (2000). Conversation. How talk can change our lives. London: The Harvill Press.

\section{Psychoanalytic Psychotherapy with Muslims in Switzerland}

Abstract: In the past, psychoanalysis was only able to gain a marginal foothold as a treatment method in Islamic societies. This marginal position is justified by the fact that Islam and psychoanalysis are in conflict with each other. Muslims would not be able to benefit from the psychoanalytic treatment approach because of their religion in the context of a society structured according to Islamic normativities. For Muslims in Switzerland, however, the initial situation is different. As an example, three treatment vignettes of Muslim migrants demonstrate that they can benefit from psychoanalytic psychotherapy. The article describes the course of psychotherapy, including the interactions between psychopathological complaints, inner-psychological conflicts and external situations in the context of individual Muslim religiosity.

Key Words: Psychoanalysis, psychoanalytic Psychotherapy, Muslims, Islam, migration, psychoanalytic case vignettes, Switzerland

\section{Psicoterapia psicoanalitica con i musulmani in Svizzera}

Riassunto: In passato, la psicoanalisi è stata in grado di acquisire una posizione marginale come metodo di trattamento nelle società islamiche. Questa posizione marginale viene giustificata dal fatto che Islam e psicoanalisi sarebbero in conflitto tra loro. Le donne musulmane* non potrebbero beneficiare dell'approccio terapeutico psicoanalitico a causa della loro religione nel contesto di una società strutturata secondo le norme islamiche. Tuttavia, la situazione di partenza è diversa per i musulmani in Svizzera. A titolo di esempio, tre disegni/ vignette di trattamento di migranti musulmani* dimostrano che possono beneficiare della psicoterapia psicoanalitica. L'articolo descrive i percorsi di psicoterapia che comprendono le interazioni tra disturbi psicopatologici, conflitti psicologici interni ed esterni nel contesto della religiosità individuale musulmana.

Parole chiave: psicoanalisi, psicoterapia psicoanalitica, musulmani, Islam, migrazione, disegni di casi psicoanalitici, Svizzera

\section{Die Autorin}

Katrin Hartmann, Dr. sc. soc., MSc, lic.phil. I, Psychoanalytische Psychotherapeutin und Soziologin, ist psychotherapeutisch im interdisziplinären Zentrum für psychische Gesundheit Sollievo sowie in eigener Praxis in Bern tätig. Sie ist Mitglied der Wissenschaftskommission der ASP und dissertierte an der Université de Genève über die Psychoanalyse im Libanon.

\section{Kontakt}

E-Mail: ka.har@bluewin.ch 